\title{
Molecular Patchy Clusters with Controllable Symmetry Breaking for Structural Engineering
}

Gang Li, ${ }^{1 \dagger}$ Zhanhui Gan, ${ }^{1 \dagger}$ Yuchu Liu, ${ }^{3}$ Shuai Wang,,${ }^{1}$ Qing-Yun Guo, ${ }^{3}$ Zhongguo Liu, ${ }^{1}$ Rui Tan, ${ }^{1}$ Dongdong Zhou, ${ }^{1}$ Deyu Kong, ${ }^{1}$ Tao Wen, ${ }^{1}$ and Xue-Hui Dong ${ }^{1,2 *}$

${ }^{1}$ South China Advanced Institute of Soft Matter Science and Technology, School of Molecular Science and Engineering, South China University of Technology, Guangzhou 510640, China

${ }^{2}$ State Key Laboratory of Luminescent Materials and Devices, South China University of Technology, Guangzhou 510640, China

${ }^{3}$ Department of Polymer Science, The University of Akron, Akron, Ohio 44325, United States

$\dagger$ These authors contribute equally

*Corresponding author: $\underline{x d o n g} @$,scut.edu.cn 


\section{Table of content}

1. Materials

2. Synthetic procedures

3. Calculation and analysis

4. Chemical structures and synthetic schemes

5. Supplementary figures and tables 


\section{Materials}

Chemicals and Solvents. The following chemicals were used as received: Tetrahydrofuran (THF, 99.5+\%, General-Reagent), Dichloromethane (DCM, 99.5+\%, General-Reagent), Petroleum Ether (PE, boiling range $60{ }^{\circ} \mathrm{C}$ to $90{ }^{\circ} \mathrm{C}$, General-Reagent), Ethyl Acetate (EA, 99.5+\%, General-Reagent), Toluene (99\%, Guangzhou chemical reagent factory), Chloroform $\left(\mathrm{CHCl}_{3}, 99+\%\right.$, Guangzhou chemical reagent factory), diethyl ether (99+\%, Guangzhou chemical reagent factory), Acetone (99+\%, Guangzhou chemical reagent factory), Methanol (MeOH, General-Reagent, 99.5+\%), Ethanol (EtOH, 99.5+\%, General-Reagen), 1-Butanethiol (98+\%, Adamas), Ammonium hydroxide solution (29\%, Adamas), 3-Mercapto-1-propanol (95+\%, 3A chemicals), 2-Mercaptoethanol (98+\%, TCI), Succinic anhydride (95+\%, TCI), Trimethylene Bromohydrin (98+\%, Adamas), 3,4-Dihydro-2H-pyran (99+\%, Adamas), $p$-toluenesulfonic acid monohydrate ( $p$-TsOH, 97+\%, Adamas), 4-Hydroxybenzoic Acid Methyl Este (99+\%, Adamas), Lithium Hydroxide (LiOH, 99+\%, Adamas), 4-Dimethylaminopyridine (DMAP, 99+\%, Adamas), $N, N^{\prime}$-diisopropylcarbodiimide (DIC, 98+\%, Aladdin), 2,2-dimethoxy-2-phenylacetophenone (DMPA, Irgacure 2959, 99\%, Sigma-Aldrich), $N, N, N^{\prime}, N^{\prime \prime}$, $N^{\prime \prime}$-pentamethyl diethylenetriamine (PMDETA, 99+\%, TCI), Potassium Carbonate $\left(\mathrm{K}_{2} \mathrm{CO}_{3}, 99.5+\%\right.$, GeneralReagent), Sodium Sulfate $\left(\mathrm{Na}_{2} \mathrm{SO}_{4}, 99+\%\right.$, General-Reagent).

Copper(I) Bromide (CuBr, 99+\%, Aladdin) was stirred in acetic acid for $24 \mathrm{~h}$, washed with ethanol and diethyl ether. 2,2-Azobis(2-Methylpropionitrile) (AIBN, 98+\%, Adamas) was recrystallized in $\mathrm{MeOH}$. OctaVinylPOSS ( $\mathrm{T}_{8}$-VPOSS, 98\%, Gileader Advanced material) was recrystallized in THF. 4(Dimethylamino) Pyridinium- $p$-toluene- Sulfonate (DPTS), ${ }_{1}^{1}$ 3-Azido-1-propanol $\left(\mathrm{N}_{3}-\mathrm{OH}\right),{ }^{2} \mathrm{~V}_{7} \mathrm{POSS}-\mathrm{COOH}^{3}$ and 4-(2-Propynyloxy) benzoic acid (yne-COOH) ${ }^{4}$ were prepared according to the reported procedures. Anhydrous solvents, including DCM, toluene, THF, were obtained with an INERT Pure Solv System (Inert Corporation, USA).

\section{Synthetic Procedures}

\subsection{Syntheses of Patchy Shells}

$\mathbf{N}_{3}$-COOH. In a $50 \mathrm{~mL}$ round-bottom flask with a magnetic stirring bar, $\mathrm{N}_{3}-\mathrm{OH}(1.01 \mathrm{~g}, 10.0 \mathrm{mmol})$, succinic anhydride (1.20 g, $12.0 \mathrm{mmol})$, DMAP (0.29 g, $2.4 \mathrm{mmol})$, and TEA (1.21 g, $12.0 \mathrm{mmol})$ were dissolved in $25 \mathrm{~mL}$ of anhydrous DCM. The mixture was then stirred for $24 \mathrm{~h}$ at room temperature. After that, a few drops of $\mathrm{HCl}(2 \mathrm{~N})$ were added into the reaction system and washed with deionized water three times. The organic layer was collected and dried with $\mathrm{Na}_{2} \mathrm{SO}_{4}$. After removing the excess solvent, the target products were obtained as colorless liquid $(1.51 \mathrm{~g}$, yield $=75.0 \%)$ after vacuum drying for $24 \mathrm{~h} .{ }^{1} \mathrm{H}$ NMR $\left(400 \mathrm{MHz}, d_{6^{-}}\right.$ 
DMSO): $\delta 12.22(\mathrm{~s}, 1 \mathrm{H}), 4.08(\mathrm{t}, 2 \mathrm{H}), 3.41(\mathrm{t}, 2 \mathrm{H}), 2.54-2.46(\mathrm{~m}, 2 \mathrm{H}), 1.86-1.79(\mathrm{~m}, 2 \mathrm{H})$.

CPOSS-OH. V V POSS-OH (3.0 g, $4.22 \mathrm{mmol})$ and DMPA (0.38 g, $1.47 \mathrm{mmol})$ were dissolved in $40 \mathrm{~mL}$ of THF. 1-Butanethiol (5.33 g, $59.08 \mathrm{mmol})$ was then added. The solution was irradiated under $365 \mathrm{~nm}$ UV light for $20 \mathrm{~min}$ at room temperature. After removing the excess solvent, the residue was purified over a silica column with a gradient eluent of EA and PE (1/30 to 1/10, v/v). CPOSS-OH was obtained as a colorless liquid (4.53 g, yield = 80.0 \%). ${ }^{1} \mathrm{H}$ NMR (400 MHz, $\left.\mathrm{CDCl}_{3}\right): \delta 3.73(\mathrm{t}, 2 \mathrm{H}), 2.73(\mathrm{t}, 2 \mathrm{H}), 2.66-2.50(\mathrm{~m}, 30 \mathrm{H}), 1.60-$ $1.52(\mathrm{~m}, 14 \mathrm{H}), 1.45-1.36(\mathrm{~m}, 14 \mathrm{H}), 1.05-0.98(\mathrm{~m}, 16 \mathrm{H}), 0.92(\mathrm{t}, 21 \mathrm{H})$.

CPOSS-N ${ }_{3}$. CPOSS-OH (4.0 g, $\left.2.98 \mathrm{mmol}\right), \mathrm{N}_{3}-\mathrm{COOH}$ (0.72 g, $\left.3.58 \mathrm{mmol}\right)$, and DMAP (0.36 g, $\left.2.98 \mathrm{mmol}\right)$ were dissolved in $30 \mathrm{~mL}$ of anhydrous DCM. DIC (0.56 g, $4.47 \mathrm{mmol})$ was then added slowly with ice bath. The mixture was stirred at room temperature overnight. The solution was diluted with DCM and washed with water three times. The organic layer was collected and dried with anhydrous $\mathrm{Na}_{2} \mathrm{SO}_{4}$. After removing the excess solvent, the residue was purified over a silica column with an mixed eluent of EA and PE (1/20, v/v). CPOSS-N $\mathrm{N}_{3}$ was obtained as a colorless liquid $(3.32 \mathrm{~g}$, yield $=73.0 \%) .{ }^{1} \mathrm{H}$ NMR $\left(400 \mathrm{MHz}, \mathrm{CDCl}_{3}\right): \delta 4.25-$ $4.18(\mathrm{~m}, 4 \mathrm{H}), 3.40(\mathrm{t}, 2 \mathrm{H}), 2.75(\mathrm{t}, 2 \mathrm{H}), 2.67-2.50(\mathrm{~m}, 34 \mathrm{H}), 1.60-1.53(\mathrm{~m}, 14 \mathrm{H}), 1.46-1.37$ (m, 14H), $1.04-$ $0.99(\mathrm{~m}, 16 \mathrm{H}), 0.92(\mathrm{t}, 21 \mathrm{H})$. MS (MALDI-TOF, $m / z)$ : Calcd for $(\mathrm{M} \cdot \mathrm{Na})^{+}\left(\mathrm{C}_{53} \mathrm{H}_{109} \mathrm{~N}_{3} \mathrm{O}_{16} \mathrm{~S}_{8} \mathrm{Si}_{8}\right): 1546.3$, Found: 1546.4.

\subsection{Syntheses of Patchy Cores}

$\mathbf{V}_{(\mathbf{8}-n)}$ POSS-nOH $(\boldsymbol{n}=\mathbf{1 , 2}, \mathbf{3})$ isomers. $\mathrm{V}_{8} \operatorname{POSS}(30.0 \mathrm{~g}, 47.39 \mathrm{mmol})$ and DMPA $(0.13 \mathrm{~g}, 0.51 \mathrm{mmol})$ were dissolved in $350 \mathrm{~mL}$ of THF. 3-Mercapto-1,2-Propanediol $(8.0 \mathrm{~g}, 86.81 \mathrm{mmol})$ was then added. The solution was irradiated under $365 \mathrm{~nm}$ UV light for $15 \mathrm{~min}$ at room temperature. After removing the excess solvent, the residue was separated over a silica column with a gradient eluent: DCM and PE (2/3, v/v) for $\mathrm{V}_{7} \mathrm{POSS}-\mathrm{OH}$ $(6.5 \mathrm{~g}$, yield $=18.91 \%)$, EA and PE $(1 / 19, \mathrm{v} / \mathrm{v})$ for $p_{2}-\mathrm{V}_{6} \mathrm{POSS}(\mathrm{OH})_{2}(0.9 \mathrm{~g}$, yield $=2.32 \%)$, EA and PE $(1 / 9$, $\mathrm{v} / \mathrm{v}$ ) for $m_{2}-\mathrm{V}_{6} \mathrm{POSS}(\mathrm{OH})_{2}(1.6 \mathrm{~g}$, yield $=4.13 \%)$, EA and $\mathrm{PE}(1 / 4, \mathrm{v} / \mathrm{v})$ for $o_{2}-\mathrm{V}_{6} \mathrm{POSS}(\mathrm{OH})_{2}(3.5 \mathrm{~g}$, yield $=$ $9.04 \%)$, THF and DCM $(1 / 15, \mathrm{v} / \mathrm{v})$ for $o_{m}-\mathrm{V}_{5} \mathrm{POSS}(\mathrm{OH})_{3}(1.8 \mathrm{~g}$, yield $=4.18 \%)$, THF and DCM $(1 / 9, \mathrm{v} / \mathrm{v})$ for $o_{3}-\mathrm{V}_{5} \mathrm{POSS}(\mathrm{OH})_{3}(2.8 \mathrm{~g}$, yield $=6.5 \%)$. V 7 POSS-OH ${ }^{1} \mathrm{H}$ NMR $\left(400 \mathrm{MHz}, \mathrm{CDCl}_{3}\right): \delta 6.14-5.87(\mathrm{~m}, 21 \mathrm{H})$, $3.75(\mathrm{t}, 2 \mathrm{H}), 2.67-2.62(\mathrm{~m}, 4 \mathrm{H}), 1.87-1.80(\mathrm{~m}, 2 \mathrm{H}), 1.10-1.05(\mathrm{~m}, 2 \mathrm{H}) . \mathrm{V}_{6} \mathrm{POSS}(\mathrm{OH})_{2}$ isomers ${ }^{1} \mathrm{H}$ NMR $(400$ $\left.\mathrm{MHz}, \mathrm{CDCl}_{3}\right): \delta 6.17-5.85(\mathrm{~m}, 18 \mathrm{H}), 3.76-3.72(\mathrm{~m}, 4 \mathrm{H}), 2.72-2.59(\mathrm{~m}, 8 \mathrm{H}), 1.88-1.81(\mathrm{~m}, 4 \mathrm{H}), 1.09-1.05(\mathrm{~m}$, 4H). $o_{2}-\mathrm{V}_{6} \mathrm{POSS}(\mathrm{OH})_{2}{ }^{29} \mathrm{Si} \mathrm{NMR}\left(500 \mathrm{MHz}, \mathrm{CDCl}_{3}\right): \delta-68.63,-80.22,-80.35 . m_{2}-\mathrm{V}_{6} \mathrm{POSS}(\mathrm{OH})_{2}{ }^{29} \mathrm{Si} \mathrm{NMR}$ $\left(500 \mathrm{MHz}, \mathrm{CDCl}_{3}\right): \delta-68.52,-80.23,-80.35,-80.48 . p_{2}-\mathrm{V}_{6} \mathrm{POSS}(\mathrm{OH})_{2}{ }^{29} \mathrm{Si} \mathrm{NMR}\left(500 \mathrm{MHz}, \mathrm{CDCl}_{3}\right): \delta-$ 68.53, -80.35. $\mathrm{V}_{5} \mathrm{POSS}(\mathrm{OH})_{3}$ isomers ${ }^{1} \mathrm{H}$ NMR $\left(400 \mathrm{MHz}, \mathrm{CDCl}_{3}\right): \delta 6.14-5.85(\mathrm{~m}, 15 \mathrm{H}), 3.78-3.73(\mathrm{~m}, 6 \mathrm{H})$, 
2.69-2.63 (m, 12H), 1.88-1.81 (m, 6H), 1.09-1.05 (m, 6H). $o_{3}-\mathrm{V}_{5} \mathrm{POSS}(\mathrm{OH})_{3}{ }^{29} \mathrm{Si} \mathrm{NMR}\left(500 \mathrm{MHz}, \mathrm{CDCl}_{3}\right)$ : $\delta$-68.61, -68.74, 80.23, -80.36, -80.48. $o_{m}-\mathrm{V}_{5} \mathrm{POSS}(\mathrm{OH}){ }_{3}{ }^{29} \mathrm{Si} \mathrm{NMR}\left(500 \mathrm{MHz}, \mathrm{CDCl}_{3}\right): \delta-68.52,-68.63,-$ $80.36,-80.49$.

General Procedures for the Preparation of $\mathbf{V}_{(8-n)} \operatorname{POSS}(\mathrm{THP})_{n}(n=1,2,3)$ isomers. Take $\boldsymbol{o}_{3^{-}}$ $\mathbf{V}_{5} \mathbf{P O S S}(\mathbf{T H P})_{3}$ as an example. $o_{3}-\mathrm{V}_{5} \mathrm{POSS}(\mathrm{OH})_{3}(5.0 \mathrm{~g}, 5.50 \mathrm{mmol})$ and $p$ - $\mathrm{TsOH}(0.57 \mathrm{mg}, 3.30 \mathrm{mmol})$ were dissolved in $50 \mathrm{~mL}$ of anhydrous DCM. 3,4-dihydro-2H-pyran (2.78 g, $33.0 \mathrm{mmol})$ was then slowly dropped into the above solution. The mixture was stirred at room temperature overnight. After TLC showed the reaction was complete, the reaction was quenched by deionized water and extracted three times. The brown layer was collected and dried with anhydrous $\mathrm{Na}_{2} \mathrm{SO}_{4}$. The crude product was purified over a silica column with an eluent of EA and PE (1/10, v/v), yielding the product as a colorless liquid $(4.47 \mathrm{~g}$, yield $=70.0 \%) .{ }^{1} \mathrm{H}$ NMR $\left(400 \mathrm{MHz}, \mathrm{CDCl}_{3}\right): \delta 6.12-5.84(\mathrm{~m}, 15 \mathrm{H}), 4.57(\mathrm{t}, 3 \mathrm{H}), 3.88-3.78(\mathrm{~m}, 6 \mathrm{H}), 3.52-3.44(\mathrm{~m}, 6 \mathrm{H}), 2.67-2.55(\mathrm{~m}$, $12 \mathrm{H}), 1.88-1.49(\mathrm{~m}, 24 \mathrm{H}), 1.07-1.01(\mathrm{~m}, 6 \mathrm{H})$. The yield of other isomers $o_{m}-\mathrm{V}_{5} \mathrm{POSS}(\mathrm{THP})_{3}$ was $72.0 \%$ and the ${ }^{1} \mathrm{H}$ NMR was similar with above of the $o_{3}-\mathrm{V}_{5} \mathrm{POSS}(\mathrm{THP})_{3}$.

V ${ }_{7}$ POSS-THP: yield $=90.0 \% .{ }^{1} \mathrm{H}$ NMR $\left(400 \mathrm{MHz}, \mathrm{CDCl}_{3}\right): \delta 6.16-5.86(\mathrm{~m}, 21 \mathrm{H}), 4.58(\mathrm{t}, 1 \mathrm{H}), 3.87-3.78$ (m, 2H), 3.51-3.46 (m, 2H), 2.68-2.60 (m, 4H), 1.89-1.49 (m, 8H), 1.10-1.05 (m, 2H).

$\mathbf{V}_{6} \mathbf{P O S S}(\mathbf{T H P})_{2}$ isomers: yield $=77.0 \%-80.0 \% .{ }^{1} \mathrm{H}$ NMR $\left(400 \mathrm{MHz}, \mathrm{CDCl}_{3}\right): \delta 6.13-5.88(\mathrm{~m}, 18 \mathrm{H}), 4.58$ $(\mathrm{t}, 2 \mathrm{H}), 3.87-3.74(\mathrm{~m}, 4 \mathrm{H}), 3.52-3.46(\mathrm{~m}, 4 \mathrm{H}), 2.7-2.60(\mathrm{~m}, 8 \mathrm{H}), 1.89-1.50(\mathrm{~m}, 16 \mathrm{H}), 1.10-1.04(\mathrm{~m}, 4 \mathrm{H})$.

General Procedures for the Preparation of $\mathbf{H}_{(8-n)} \operatorname{POSS}(\mathrm{THP})_{n}(n=1,2,3)$ isomers. Take $\boldsymbol{o}_{3^{-}}$ $\mathbf{H}_{5}$ POSS(THP) $)_{3}$ as an example. $o_{3}-\mathrm{V}_{5} \mathrm{POSS}(\mathrm{THP})_{3}(4.0 \mathrm{~g}, 3.44 \mathrm{mmol})$, 3-Mercapto-1,2-Propanediol (3.17 g, $34.43 \mathrm{mmol})$, and AIBN (0.28 g, $1.72 \mathrm{mmol})$ were dissolved in $250 \mathrm{~mL}$ of dried toluene under pure nitrogen flow. The solution was stirred at $75^{\circ} \mathrm{C}$ for 24 hours and separated over a silica column with a gradient eluent of THF and DCM (1/19 to 1/1.5, v/v), yielding the product as a light-yellow liquid (3.35 g, yield $=60.0 \%)$. ${ }^{1} \mathrm{H}$ NMR (400 MHz, $d_{6}$-Acetone): 4.53 (t, 3H), 3.78-3.71 (m, 6H), 3.66-3.56 (m, 10H), 3.43-3.38 (m, 6H), 2.65-2.56 (m, 32H), 1.82-1.44 (m, 34H), 1.04-1.01 (m, 16H). The yield of other isomers $o_{m}-\mathrm{H}_{5} \mathrm{POSS}(\mathrm{THP})_{3}$ was $62 \%$ and the ${ }^{1} \mathrm{H}$ NMR was similar with above of the $o_{3}-\mathrm{H}_{5} \mathrm{POSS}(\mathrm{THP})_{3}$.

H7POSS-THP: yield $=71.0 \%$. ${ }^{1} \mathrm{H}$ NMR (400 MHz, $d_{6}$-Acetone): $\delta 4.59(\mathrm{t}, 1 \mathrm{H}), 3.79-3.71(\mathrm{~m}, 2 \mathrm{H}), 3.68$ $3.60(\mathrm{~m}, 14 \mathrm{H}), 3.49-3.43(\mathrm{~m}, 2 \mathrm{H}), 2.70-2.61(\mathrm{~m}, 32 \mathrm{H}), 1.81-1.48(\mathrm{~m}, 22 \mathrm{H}), 1.1-1.05(\mathrm{~m}, 16 \mathrm{H})$.

$\mathbf{H}_{6}$ POSS(THP) $)_{2}$ isomers: yield $=65.0 \%-68.0 \%$. ${ }^{1} \mathrm{H}$ NMR (400 MHz, $d_{6}$-Acetone): $\delta 4.59$ (t, 2H), 3.84$3.77(\mathrm{~m}, 4 \mathrm{H}), 3.67-3.63(\mathrm{~m}, 12 \mathrm{H}), 3.49-3.44(\mathrm{~m}, 4 \mathrm{H}), 2.75-2.59(\mathrm{~m}, 32 \mathrm{H}), 1.87-1.49(\mathrm{~m}, 28 \mathrm{H}), 1.10-1.06(\mathrm{~m}$, 
$16 \mathrm{H})$.

General Procedures for the Preparation of $(\mathrm{yne})_{(8-n)}(\operatorname{POSS})(\mathrm{OH})_{n}(n=1,2,3)$ isomers. Take the $o_{3^{-}}$ (yne $)_{5}(\mathbf{P O S S})(\mathbf{O H})_{3}$ as an example. $o_{3}-\mathrm{H}_{5} \mathrm{POSS}(\mathrm{THP})_{3}(3.0 \mathrm{~g}, 1.82 \mathrm{mmol})$, 4-(2-Propynyloxy) benzoic acid (2.41 g, $13.65 \mathrm{mmol})$, and DMAP (1.67 g, $13.65 \mathrm{mmol})$ were dissolved in $100 \mathrm{~mL}$ of dried THF. DIC (1.72 g, $13.65 \mathrm{mmol}$ ) was then slowly dropped into the above solution with an ice bath. The mixture was stirred at room temperature overnight. The solution was diluted with DCM and washed with deionized water three times. The organic layer was collected and dried with anhydrous $\mathrm{Na}_{2} \mathrm{SO}_{4}$. After removing the excess solvent, the crude product was separated over a silica column with a gradient eluent of THF and DCM $(0 / 1$ to $1 / 19, \mathrm{v} / \mathrm{v})$, yielding with a light-yellow liquid. Then, $o_{3}$-(yne $)_{5}(\mathrm{POSS})(\mathrm{THP})_{3}$ was dissolved in $60 \mathrm{~mL}$ mixed solvent of DCM and $\mathrm{MeOH}(1 / 1, \mathrm{v} / \mathrm{v})$ for the deprotection of THP. The $p$-toluene sulfonic acid monohydrate was added per hour until TLC showed the reaction had completed. The reaction was quenched by ammonium hydroxide and extracted with DCM. After removing the excess solvent, the residue was separated over a silica column with an mixed eluent of THF and DCM $(1 / 19, \mathrm{v} / \mathrm{v})$, yielding the product as a colorless viscous liquid $(2.75 \mathrm{~g}$, yield $=70.0 \%) .{ }^{1} \mathrm{H}$ NMR $\left(400 \mathrm{MHz}, \mathrm{CDCl}_{3}\right): \delta 8.01-7.98(\mathrm{~m}, 10 \mathrm{H}), 7.01-6.97(\mathrm{~m}, 10 \mathrm{H}), 4.75-4.73(\mathrm{~m}, 10 \mathrm{H})$, 4.40-4.35 (m, 10H), 3.74-3.71 (m, 6H), 2.68-2.61 (m, 32H), $2.55(\mathrm{~s}, 5 \mathrm{H}), 2.06-1.99(\mathrm{~m}, 10 \mathrm{H}), 1.86-1.79(\mathrm{~m}$, $6 \mathrm{H})$, 1.06-1.02 (m, 16H). MS (MALDI-TOF, $m / z)$ : Calcd for $(\mathrm{M} \cdot \mathrm{Na})^{+}\left(\mathrm{C}_{90} \mathrm{H}_{118} \mathrm{O}_{30} \mathrm{~S}_{8} \mathrm{Si}_{8}\right)$ : 2181.3, Found: 2181.5. The yield of other isomers $\boldsymbol{o}_{\boldsymbol{m}}-(\mathbf{y n e})_{5}(\mathbf{P O S S})(\mathbf{O H})_{3}$ was $68.0 \%$ and the ${ }^{1} \mathrm{H}$ NMR was similar with above of the $o_{3}-(\mathrm{yne})_{5}(\mathrm{POSS})(\mathrm{OH})_{3}$. MS (MALDI-TOF, $\left.\mathrm{m} / \mathrm{z}\right)$ : Calcd for $(\mathrm{M} \cdot \mathrm{Na})^{+}\left(\mathrm{C}_{90} \mathrm{H}_{118} \mathrm{O}_{30} \mathrm{~S}_{8} \mathrm{Si}_{8}\right): 2181.3$, Found: 2181.8 .

(yne) $)_{7}(\mathbf{P O S S})-\mathrm{OH}$ yield $=85.0 \%$ \%. ${ }^{1} \mathrm{H}$ NMR $\left(400 \mathrm{MHz}, \mathrm{CDCl}_{3}\right): \delta 8.0-7.97(\mathrm{~m}, 14 \mathrm{H}), 6.99-6.97(\mathrm{~m}, 14 \mathrm{H})$, 4.73-4.71 (m, 14H), 4.38-4.34 (m, 14H), 3.73-3.69 (m, 2H), 2.66-2.59 (m, 32H), 2.55 (s, 7H), 2.03-1.99 (m, 14H), 1.83-1.77 (m, 2H), 1.05-1.01 (m, 16H). MS (MALDI-TOF, $m / z)$ : Calcd for $(\mathrm{M} \cdot \mathrm{Na})^{+}\left(\mathrm{C}_{110} \mathrm{H}_{130} \mathrm{O}_{34} \mathrm{~S}_{8} \mathrm{Si}_{8}\right)$ : 2474.4, Found: 2474.5.

$(\text { yne })_{6}(\mathbf{P O S S})(\mathbf{O H})_{2}$ isomers yield $=72.0 \%-75.0 \%$. ${ }^{1} \mathrm{H}$ NMR $\left(400 \mathrm{MHz}, \mathrm{CDCl}_{3}\right): \delta 8.0-7.97(\mathrm{~m}, 12 \mathrm{H})$, 7.08-6.97 (m, 12H), 4.74-4.72 (m, 12H), 4.38-4.34 (m, 12H), 3.73-3.70 (m, 4H), 2.67-2.61 (m, 32H), 2.56$2.54(\mathrm{~m}, 6 \mathrm{H}), 2.05-1.97(\mathrm{~m}, 12 \mathrm{H}), 1.85-1.78(\mathrm{~m}, 4 \mathrm{H}), 1.06-1.01(\mathrm{~m}, 16 \mathrm{H})$. MS (MALDI-TOF, $m / z)$ : (yne $)_{6}(\mathrm{POSS})(\mathrm{OH})_{2}$ isomers Calcd for $(\mathrm{M} \cdot \mathrm{Na})^{+}\left(\mathrm{C}_{100} \mathrm{H}_{124} \mathrm{O}_{32} \mathrm{~S}_{8} \mathrm{Si}_{8}\right)$ : 2339.4, $\boldsymbol{o}_{2}-(\mathbf{y n e})_{6}(\mathbf{P O S S})(\mathbf{O H})_{2}$ Found: 2339.5, $\boldsymbol{m}_{2}-(\mathbf{y n e})_{6}(\mathbf{P O S S})(\mathbf{O H})_{2}$ Found: 2339.8, $\boldsymbol{p}_{2}-(\mathbf{y n e})_{6}(\mathbf{P O S S})(\mathbf{O H})_{2}$ Found: 2339.8.

\subsection{Syntheses of Patchy Clusters}

General Procedures for the Preparation of $(\text { yne })_{(8-n)}($ POSS $)(\text { VPOSS })_{n}(n=1,2,3)$ isomers. Take $o_{3^{-}}$ 
(yne $)_{5}($ POSS $)(\text { VPOSS })_{3}$ as an example. $o_{3}-(\mathrm{yne})_{5}(\mathrm{POSS})(\mathrm{OH})_{3}(0.30 \mathrm{~g}, 0.13 \mathrm{mmol}), \mathrm{V}_{7} \mathrm{POSS}-\mathrm{COOH}(0.49$ g, $0.60 \mathrm{mmol}$ ), and DPTS (134.0 mg, $0.60 \mathrm{mmol}$ ) were dissolved in $20 \mathrm{~mL}$ of dried DCM. DIC (76.0 mg, 0.60 mmol) was then slowly dropped into the above solution with an ice bath. The mixture was stirred at room temperature overnight. After removing the excess solvent, the crude product was separated over a silica column with an mixed eluent of EA and PE $(1 / 4, \mathrm{v} / \mathrm{v})$, yielding the product as a white solid $(0.39 \mathrm{~g}$, yield $=$ $65.0 \%$ ). ${ }^{1} \mathrm{H}$ NMR (400 MHz, $\left.\mathrm{CDCl}_{3}\right): \delta 8.0-7.97(\mathrm{~m}, 10 \mathrm{H}), 7.0-6.97(\mathrm{~m}, 10 \mathrm{H}), 6.15-5.86(\mathrm{~m}, 63 \mathrm{H}), 4.74-4.72$ (m, 10H), 4.39-4.34 (m, 10H), $4.16(\mathrm{t}, 12 \mathrm{H}), 2.67-2.55(\mathrm{~m}, 61 \mathrm{H}), 2.06-1.97(\mathrm{~m}, 10 \mathrm{H}), 1.92-1.83(\mathrm{~m}, 12 \mathrm{H})$, 1.08-1.0 (m, 22H). MS (MALDI-TOF, $m / z)$ : Calcd for $(\mathrm{M} \cdot \mathrm{Na})^{+}\left(\mathrm{C}_{159} \mathrm{H}_{220} \mathrm{~F}_{119} \mathrm{O}_{75} \mathrm{~S}_{11} \mathrm{Si}_{32}\right)$ : 4605.8, Found: 4606.0. The yield of other isomers $\boldsymbol{o}_{\boldsymbol{m}}$-(yne $)_{5}(\mathbf{P O S S})(\text { VPOSS })_{3}$ was $64.0 \%$ and the ${ }^{1} \mathrm{H}$ NMR was similar with above of the $o_{3}-(\mathrm{yne})_{5}(\mathrm{POSS})(\mathrm{VPOSS})_{3}$. MS (MALDI-TOF, $\left.\mathrm{m} / \mathrm{z}\right)$ : Calcd for $(\mathrm{M} \cdot \mathrm{Na})^{+}\left(\mathrm{C}_{159} \mathrm{H}_{220} \mathrm{~F}_{119} \mathrm{O}_{75} \mathrm{~S}_{11} \mathrm{Si}_{32}\right)$ : 4605.8, Found: 4606.1.

(yne) $)_{7}\left(\right.$ POSS)-VPOSS yield = 82.0 \%. ${ }^{1} \mathrm{H}$ NMR (400 MHz, $\left.\mathrm{CDCl}_{3}\right): \delta$ 8.0-7.97 (m, 14H), 6.99-6.97 (m, 14H), 6.12-5.90 (m, 21H), $4.73(\mathrm{t}, 14 \mathrm{H}), 4.37-4.34(\mathrm{~m}, 14 \mathrm{H}), 4.17-4.13(\mathrm{~m}, 4 \mathrm{H}), 2.66-2.53(\mathrm{~m}, 47 \mathrm{H}), 2.04-1.97(\mathrm{~m}$, 14H), 1.91-1.84 (m, 4H), 1.08-1.0 (m, 18H). MS (MALDI-TOF, $m / z)$ : Calcd for $(\mathrm{M} \cdot \mathrm{Na})^{+}\left(\mathrm{C}_{133} \mathrm{H}_{164} \mathrm{O}_{49} \mathrm{~S}_{9} \mathrm{Si}_{16}\right)$ : 3303.4, Found: 3303.7.

(yne) $)_{6}(\text { POSS)(VPOSS })_{2}$ isomers yield $=75.0 \%-78.0 \% .{ }^{1} \mathrm{H} \mathrm{NMR}\left(400 \mathrm{MHz}, \mathrm{CDCl}_{3}\right): \delta$ 8.0-7.97 (m, $\left.12 \mathrm{H}\right)$, 6.99-6.97 (m, 12H), 6.13-5.84 (m, 42H), 4.74-4.72 (m, 12H), 4.38-4.37 (m, 12H), $4.16(\mathrm{t}, 8 \mathrm{H}), 2.66-2.55(\mathrm{~m}$, 54H), 2.04-1.97 (m, 12H), 1.91-1.84 (m, 8H), 1.08-1.0 (m, 20H). MS (MALDI-TOF, $m / z)$ : (yne $)_{6}(\text { POSS)(VPOSS })_{2}$ Calcd for $(\mathrm{M} \cdot \mathrm{Na})^{+}\left(\mathrm{C}_{146} \mathrm{H}_{192} \mathrm{O}_{62} \mathrm{~S}_{10} \mathrm{Si}_{24}\right)$ : 3951.3, $\boldsymbol{o}_{2}-(\mathbf{y n e})_{6}(\text { POSS)(VPOSS })_{2}$ Found: 3951.7, $\boldsymbol{m}_{2}-(\mathbf{y n e})_{6}(\text { POSS)(VPOSS) })_{2}$ Found: 3952.1, $\boldsymbol{p}_{2}-(\mathbf{y n e})_{6}(\text { POSS)(VPOSS) })_{2}$ Found: 3952.1.

General Procedures for the Preparation of $\operatorname{CPOSS}_{(8-n)}(\operatorname{POSS})(\text { VPOSS })_{n}(n=1,2,3)$ isomers. Take the $\boldsymbol{o}_{3}-(\mathbf{C P O S S})_{5}(\mathrm{POSS})(\text { VPOSS })_{3}$ as an example. $o_{3}$-(yne) $)_{5}(\mathrm{POSS})(\mathrm{VPOSS})_{3}(92.1 \mathrm{mg}, 0.02 \mathrm{mmol}), \mathrm{CPOSS}_{3}$ $(0.50 \mathrm{~g}, 0.15 \mathrm{mmol})$, and $\mathrm{CuBr}(22.0 \mathrm{mg}, 0.15 \mathrm{mmol})$ were dissolved in $15 \mathrm{~mL}$ of THF in a Schlenk flask. PMDETA (27.0 mg, $0.15 \mathrm{mmol}$ ) was added when the flask was degassed by three freeze-vacuum-thaw cycles. After that, the flask was stirred at room temperature for 48 hours. The solution was passed through a silica gel column with $\mathrm{CHCl}_{3}$ as eluent to remove copper and purified by Preparative Gel Permeation Chromatography with THF as eluent. The product was obtained as viscous liquid $(0.15 \mathrm{~g}$, yield $=62.0 \%)$ after vacuum drying for 24 h. ${ }^{1} \mathrm{H}$ NMR (400 MHz, $\left.\mathrm{CDCl}_{3}\right): \delta 7.99-7.96(\mathrm{~m}, 10 \mathrm{H}), 7.70(\mathrm{~s}, 5 \mathrm{H}), 7.01-6.99(\mathrm{~m}, 10 \mathrm{H}), 6.12-5.86(\mathrm{~m}$, $63 \mathrm{H}), 5.27-5.19(\mathrm{~m}, 10 \mathrm{H}), 4.47(\mathrm{t}, 10 \mathrm{H}), 4.38-4.33(\mathrm{~m}, 10 \mathrm{H}), 4.23(\mathrm{t}, 10 \mathrm{H}), 4.15(\mathrm{t}, 22 \mathrm{H}), 2.77-2.50(\mathrm{~m}, 236 \mathrm{H})$, 2.36-2.19 (m, 10H), 2.08-1.96 (m, 10H), 1.92-1.83 (m, 12H), 1.61-1.33 (m, 140H), 1.04-0.86 (m, 207H). The 
yield of other isomers $\boldsymbol{o}_{\boldsymbol{m}}-(\mathbf{C P O S S})_{\mathbf{5}}(\mathbf{P O S S})\left(\right.$ VPOSS) 3 was $62.0 \%$ and the ${ }^{1} \mathrm{H}$ NMR was similar with above of the $\boldsymbol{o}_{3}-(\text { CPOSS })_{5}(\text { POSS)(VPOSS) })_{3}$. MS (MALDI-TOF, $\left.m / z\right)$ : (CPOSS) $)_{5}(\text { POSS)(VPOSS })_{3}$ isomers Calcd for $(\mathrm{M} \cdot \mathrm{Na})^{+}\left(\mathrm{C}_{464} \mathrm{H}_{846} \mathrm{~N}_{18} \mathrm{O}_{156} \mathrm{~S}_{58} \mathrm{Si}_{72}\right)$ : 12233.9, $\boldsymbol{o}_{3}$-(CPOSS) $)_{5}$ (POSS)(VPOSS) $)_{3}$ Found: 12233.1.

$(\text { CPOSS })_{7}($ POSS $)-V P O S S$ yield $=53.0 \% .{ }^{1} \mathrm{H}$ NMR $\left(400 \mathrm{MHz}, \mathrm{CDCl}_{3}\right): \delta 7.98-7.96(\mathrm{~m}, 14 \mathrm{H}), 7.70(\mathrm{~s}, 7 \mathrm{H})$, 7.0-6.98 (m, 14H), 6.12-5.86 (m, 21H), 5.24-5.21 (m, 14H), $4.47(\mathrm{t}, 14 \mathrm{H}), 4.39-4.31(\mathrm{~m}, 14 \mathrm{H}), 4.23(\mathrm{t}, 14 \mathrm{H})$, $4.15(\mathrm{t}, 18 \mathrm{H}), 2.77-2.50(\mathrm{~m}, 292 \mathrm{H}), 2.31-2.23(\mathrm{~m}, 14 \mathrm{H}), 2.04-1.97(\mathrm{~m}, 14 \mathrm{H}), 1.91-1.85(\mathrm{~m}, 4 \mathrm{H}), 1.61-1.36(\mathrm{~m}$, 196H), 1.04-0.9 (m, 277H). MS (MALDI-TOF, $m / z)$ : Calcd for $(\mathrm{M} \cdot \mathrm{Na})^{+}\left(\mathrm{C}_{504} \mathrm{H}_{927} \mathrm{~N}_{21} \mathrm{O}_{161} \mathrm{~S}_{65} \mathrm{Si}_{72}\right)$ : 13967.0, Found: 13967.3.

$(\mathbf{C P O S S})_{6}(\mathbf{P O S S})(\text { VPOSS })_{2}$ isomers yield $=57.0 \%-62.0 \% .{ }^{1} \mathrm{H}$ NMR $\left(400 \mathrm{MHz}, \mathrm{CDCl}_{3}\right): \delta 7.98-7.96(\mathrm{~m}$, 12H), $7.70(\mathrm{~s}, 6 \mathrm{H}), 7.0-6.97(\mathrm{~m}, 12 \mathrm{H}), 6.12-5.85(\mathrm{~m}, 42 \mathrm{H}), 5.23-5.2(\mathrm{~m}, 12 \mathrm{H}), 4.47(\mathrm{t}, 12 \mathrm{H}), 4.37-4.32(\mathrm{~m}$, $12 \mathrm{H}), 4.23(\mathrm{t}, 12 \mathrm{H}), 4.15(\mathrm{t}, 20 \mathrm{H}), 2.77-2.49(\mathrm{~m}, 264 \mathrm{H}), 2.31-2.24(\mathrm{~m}, 12 \mathrm{H}), 2.04-1.95(\mathrm{~m}, 12 \mathrm{H}), 1.89-1.84$ (m, 8H), 1.59-1.36 (m, 168H), 1.03-0.9 (m, 242H). MS (MALDI-TOF, m/z): (CPOSS) ${ }_{6}(\boldsymbol{P O S S})(\boldsymbol{V P O S S})_{2}$ isomers Calcd for $(\mathrm{M} \cdot \mathrm{Na})^{+}\left(\mathrm{C}_{464} \mathrm{H}_{846} \mathrm{~N}_{18} \mathrm{O}_{158} \mathrm{~S}_{58} \mathrm{Si}_{72}\right)$ : 13103.4, o $-(\text { CPOSS })_{6}$ (POSS)(VPOSS) ${ }_{2}$ Found: 13103.6.

General Procedures for the Preparation of $\operatorname{CPOSS}_{(8-n)}(\operatorname{POSS})(\mathrm{DPOSS})_{n}(n=1,2,3)$ isomers. Take $\boldsymbol{o}_{3^{-}}$ (CPOSS $_{5}($ POSS $)(D P O S S)_{3}$ as an example. $o_{3}-(\mathrm{CPOSS})_{5}(\mathrm{POSS})(\mathrm{VPOSS})_{3}(0.16 \mathrm{~g}, 0.013 \mathrm{mmol})$ and DMPA (3.0 mg, $0.01 \mathrm{mmol})$ were dissolved in $5 \mathrm{~mL}$ THF. 3-Mercapto-1,2-Propanediol (60.0 mg, $0.55 \mathrm{mmol}$ ) was then added. The solution was irradiated under $365 \mathrm{~nm}$ UV light for $20 \mathrm{~min}$ at room temperature. After removing the excess solvent, the solution was purified by precipitated into $\mathrm{MeOH}$ three times. The product was obtained as colorless viscous liquid $(0.15 \mathrm{~g}$, yield $=83.0 \%) .{ }^{1} \mathrm{H}$ NMR $\left(400 \mathrm{MHz}, \mathrm{CDCl}_{3}\right): \delta 7.98-7.96$ $(\mathrm{m}, 10 \mathrm{H}), 7.71(\mathrm{~s}, 5 \mathrm{H}), 7.01-6.98(\mathrm{~m}, 10 \mathrm{H}), 5.23-5.19(\mathrm{~m}, 10 \mathrm{H}), 4.48(\mathrm{t}, 10 \mathrm{H}), 4.41-4.34(\mathrm{~m}, 10 \mathrm{H}), 4.24(\mathrm{t}$, $10 \mathrm{H}), 4.16(\mathrm{t}, 22 \mathrm{H}), 3.82-3.58(\mathrm{~m}, 63 \mathrm{H}), 2.77-2.50(\mathrm{~m}, 320 \mathrm{H}), 2.32-2.25(\mathrm{~m}, 10 \mathrm{H}), 2.05-1.99(\mathrm{~m}, 10 \mathrm{H}), 1.96-$ $1.82(\mathrm{~m}, 12 \mathrm{H}), 1.60-1.39(\mathrm{~m}, 140 \mathrm{H}), 1.04-0.91(\mathrm{~m}, 249 \mathrm{H})$. The yield of other isomers $\boldsymbol{o}_{\boldsymbol{m}^{-}}$ (CPOSS $_{5}(\text { POSS)(DPOSS) })_{3}$ was $85.0 \%$ and the ${ }^{1} \mathrm{H}$ NMR was similar with above of the $\boldsymbol{o}_{3^{-}}$ $(\text { CPOSS })_{5}($ POSS $)(D P O S S)_{3}$.

(CPOSS) 7 (POSS)-DPOSS yield = 90.0 \%. ${ }^{1} \mathrm{H}$ NMR (400 MHz, $\left.\mathrm{CDCl}_{3}\right): \delta$ 7.99-7.96 (m, 14H), $7.70(\mathrm{~s}, 7 \mathrm{H})$, 7.05-6.99 (m, 14H), 5.23-5.19 (m, 14H), $4.47(\mathrm{t}, 14 \mathrm{H}), 4.38-4.33(\mathrm{~m}, 14 \mathrm{H}), 4.23(\mathrm{t}, 14 \mathrm{H}), 4.15(\mathrm{t}, 18 \mathrm{H}), 3.80-$ $3.57(\mathrm{~m}, 21 \mathrm{H}), 2.76-2.50(\mathrm{~m}, 320 \mathrm{H}), 2.29-2.26(\mathrm{~m}, 14 \mathrm{H}), 2.04-1.99(\mathrm{~m}, 14 \mathrm{H}), 1.94-1.88(\mathrm{~m}, 4 \mathrm{H}), 1.58-1.33$ (m, 196H), 1.03-0.90 (m, 291H).

$(\text { CPOSS })_{6}($ POSS $)(\text { DPOSS })_{2}$ isomers yield $=86.0 \%-89.0 \% .{ }^{1} \mathrm{H}$ NMR $\left(400 \mathrm{MHz}, \mathrm{CDCl}_{3}\right):{ }^{1} \mathrm{H} \mathrm{NMR}(400$ 
$\left.\mathrm{MHz}, \mathrm{CDCl}_{3}\right): \delta$ 7.98-7.96 (m, 12H), $7.72(\mathrm{~s}, 6 \mathrm{H}), 7.05-7.01(\mathrm{~m}, 12 \mathrm{H}), 5.24-5.18(\mathrm{~m}, 12 \mathrm{H}), 4.48(\mathrm{t}, 12 \mathrm{H})$, 4.36-4.31 (m, 12H), $4.23(\mathrm{t}, 12 \mathrm{H}), 4.15(\mathrm{t}, 20 \mathrm{H}), 3.80-3.56(\mathrm{~m}, 42 \mathrm{H}), 2.77-2.50(\mathrm{~m}, 320 \mathrm{H}), 2.29-2.27(\mathrm{~m}$, 12H), 2.07-1.96 (m, 12H), 1.94-1.85 (m, 8H), 1.57-1.39 (m, 168H), 1.03-0.91 (m, 270H). 


\section{Calculation and Analysis}

Average diameter of the column in the HEX phases (D) can be determined using eqn (1)

$$
\mathrm{D}=2 \mathrm{~d} / \sqrt{3} \#(1)
$$

where $d$ is the domain size of first peak in the SAXS patterns.

Average number of molecules per hexagonal column lattices $(\mu)$ can be determined using eqn (2)

$$
\mu=\frac{\rho V N_{A}}{M}=\frac{\sqrt{3} N_{A} D^{2} h \rho}{2 M} \#(2)
$$

where $\mathrm{D}$ is the average columnar diameter of the HEX phases, $h$ is the average height of the column stratum (the size of DPOSS are about $1.2 \mathrm{~nm}$ ), $\rho$ is the density of the sample, $M$ is the molecular weight of the sample and $N_{A}$ is the Avogadro's number $\left(N_{A}=6.022 \times 10^{23} \mathrm{~mol}^{-1}\right)$.

Cubic lattice parameters in DG phase (a) can be determined using eqn (3)

$$
a=\sqrt{6} d \#(3)
$$

where $d$ is the domain size of first peak in the SAXS patterns.

Average spherical diameter of the motif in F-K $\sigma$ phase (D) can be determined using eqn (4)

$$
\mathrm{D}=\sqrt[3]{V / 5 \pi} \#(4)
$$

where $V$ is the size of one sigma phase lattice $\left(33.59 \times 17.77 \mathrm{~nm}^{3}\right)$.

Average numbers of molecules per supramolecular sphere in F-K $\sigma$ phase $(\mu)$ can be determined using eqn (5)

$$
\mu=\frac{\rho V N_{A}}{30 M} \#(5)
$$

where $V$ is the size of one sigma phase lattice $\left(33.59 \times 17.77 \mathrm{~nm}^{3}\right), \rho$ is the density of the sample, 30 is the number of spheres in one sigma lattice, $M$ is molecular weight of sample and $N_{A}$ is the Avogadro's number $\left(N_{A}=6.022 \times 10^{23} \mathrm{~mol}^{-1}\right)$. 


\section{Chemical structures and synthetic schemes}

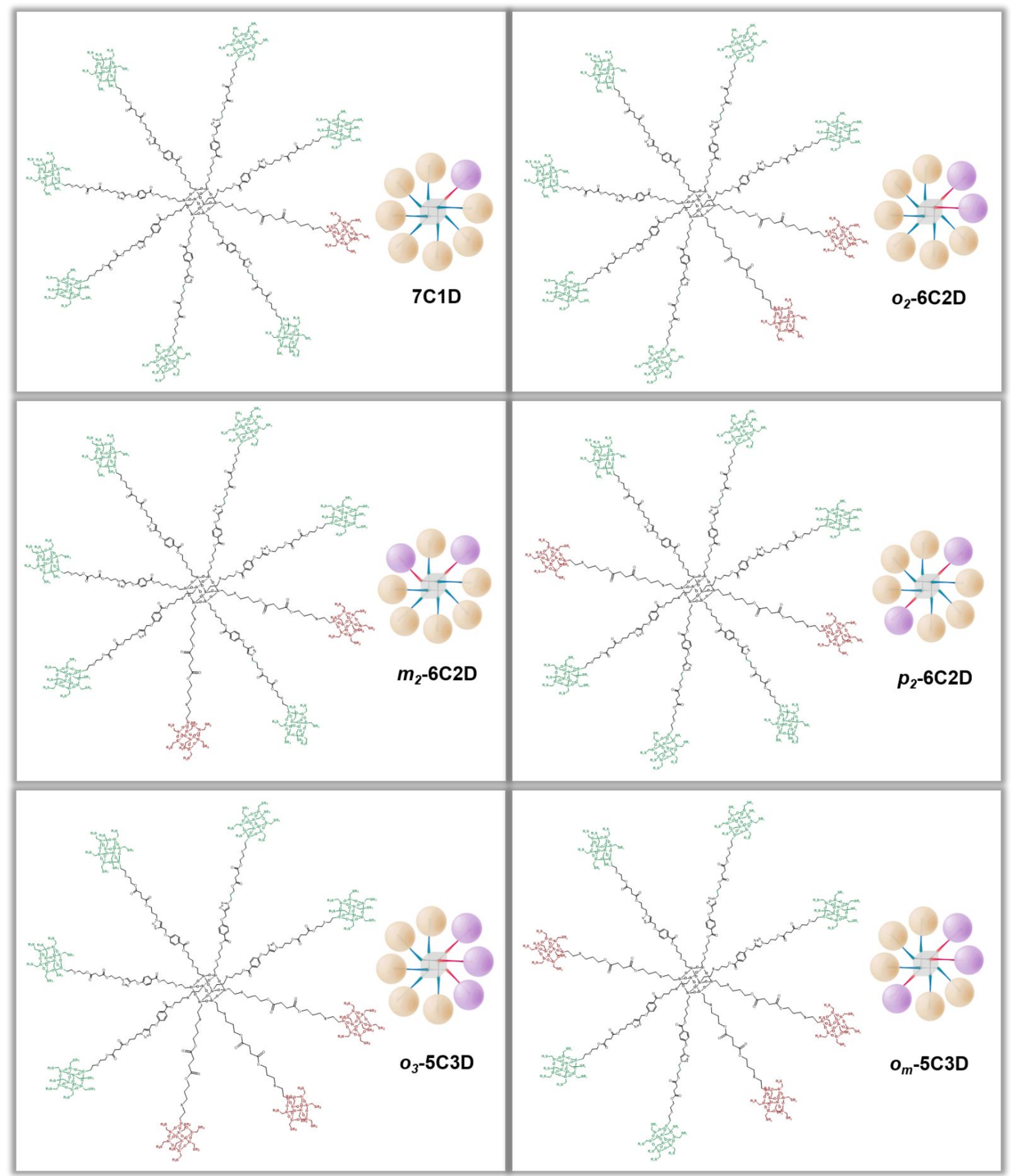

Scheme S1. Chemical structures of molecular patchy clusters. $R_{1}$ represents $n$-butyl ligands and $R_{2}$ represents hydroxyl groups. 


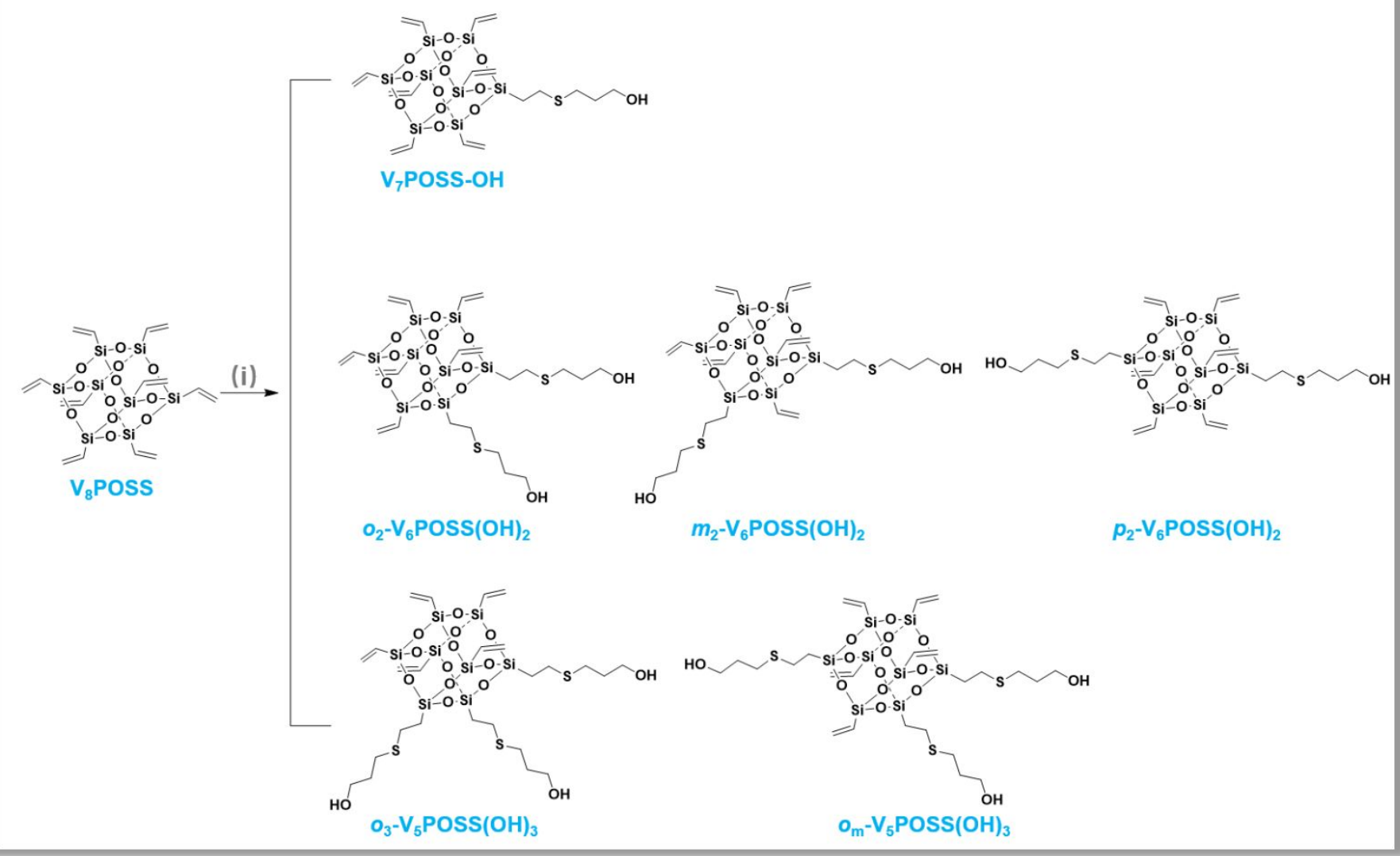

Scheme S2. Syntheses of the core block $\left(\mathrm{V}_{(8-n)} \mathrm{POSS}(\mathrm{OH})_{n}, n=1\right.$, 2, and 3): (i) 3-mercapto-1-propanol, DMPA, 365 nm UV light.

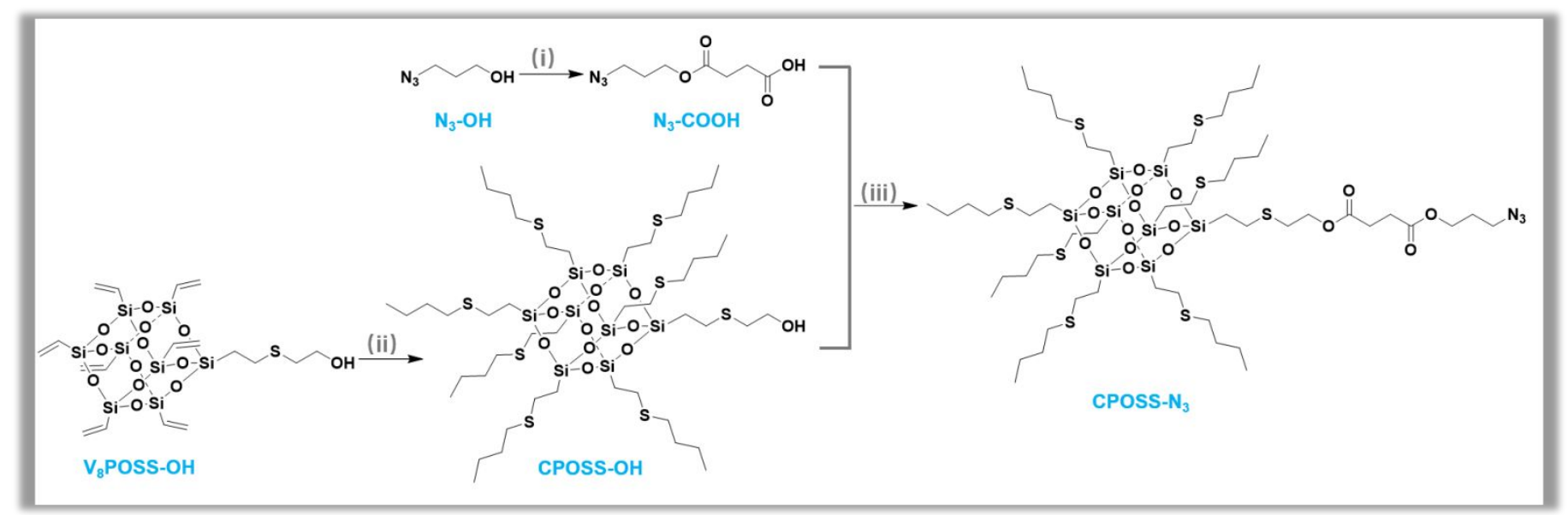

Scheme S3. Syntheses of the peripheric units: (i) succinic anhydride, DMAP and TEA, 75.0\%; (ii) 1-butanethiol, DMPA, 365 nm UV light, $80.0 \%$; (iii) DMAP and DIC, $73.0 \%$. 


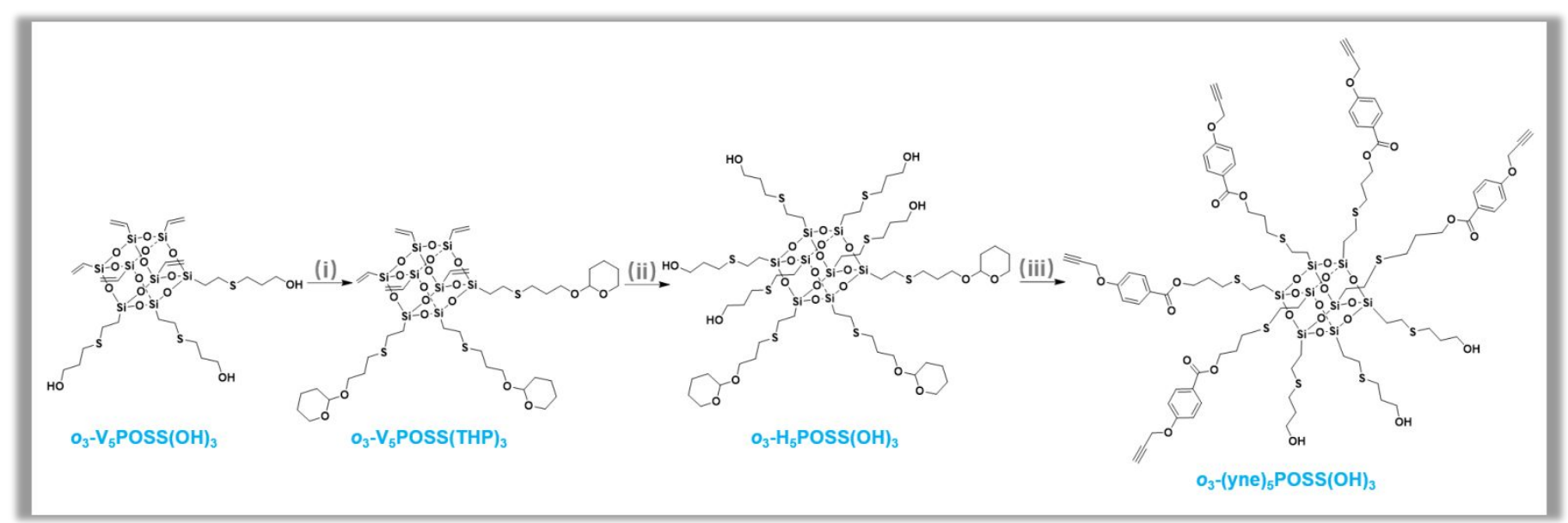

Scheme S4. Syntheses of the asymmetric core $\left(o_{3}-(\mathrm{yne})_{5}(\mathrm{POSS})(\mathrm{OH})_{3}\right)$ : (i) 3,4-dihydro-2H-pyran, $p$-TsOH, $70.0 \%$; (ii) 3-mercapto-1-propanol, AIBN, $70{ }^{\circ} \mathrm{C}, 24 \mathrm{~h}, 60.0 \%$; (iii) 4-(2-propynyloxy) benzoic acid, DMAP, DIC, $p$-TsOH, $\mathrm{DCM} / \mathrm{MeOH}(1 / 1, \mathrm{v} / \mathrm{v}), 70.0 \%$.

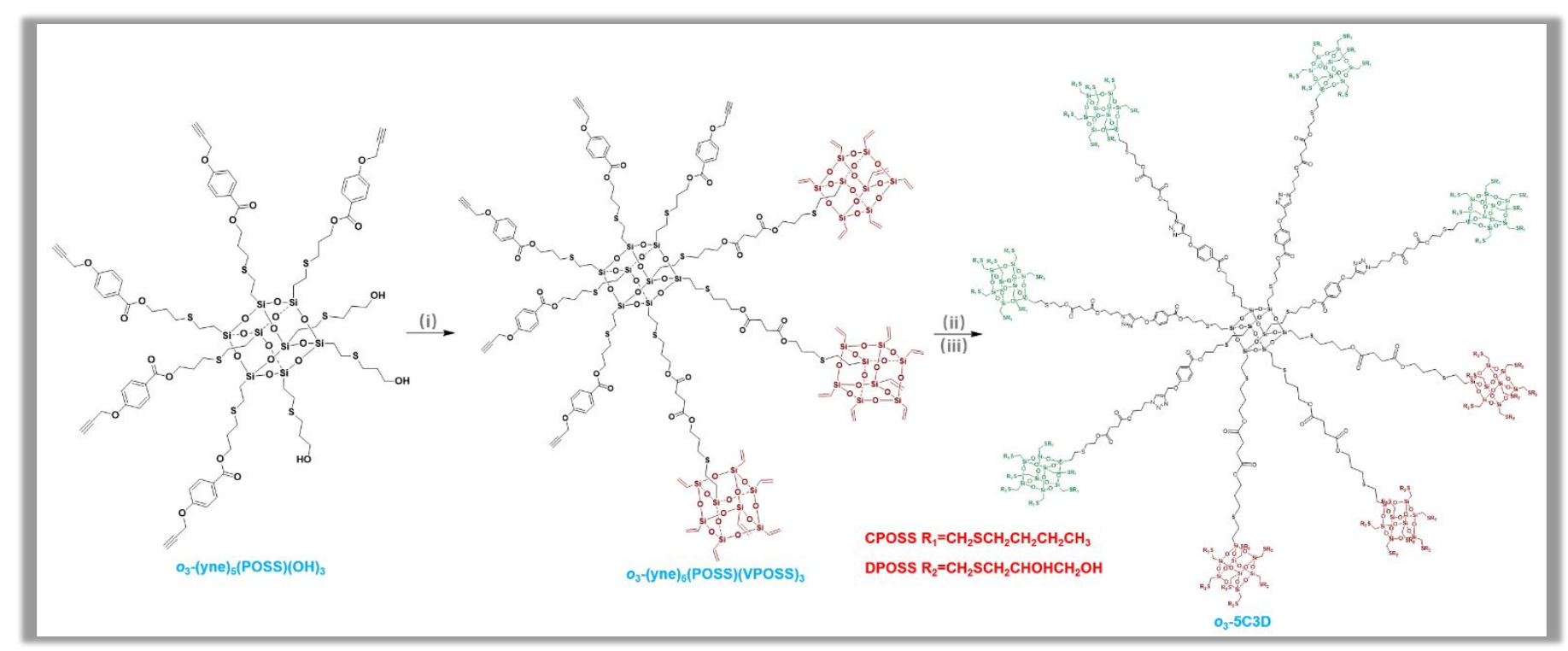

Scheme S5. Synthetic procedures for the asymmetric patchy clusters $o_{3}-5 \mathrm{C} 3 \mathrm{D}$ : (i) VPOSS-COOH, DPTS, DIC, DCM, $65.0 \%$; (ii) CPOSS-N3, PMDETA, CuBr, THF, 48h, 62.0\%; (iii) 3-mercapto-1-propanol, DMPA, 365 nm UV light, $83.0 \%$. 


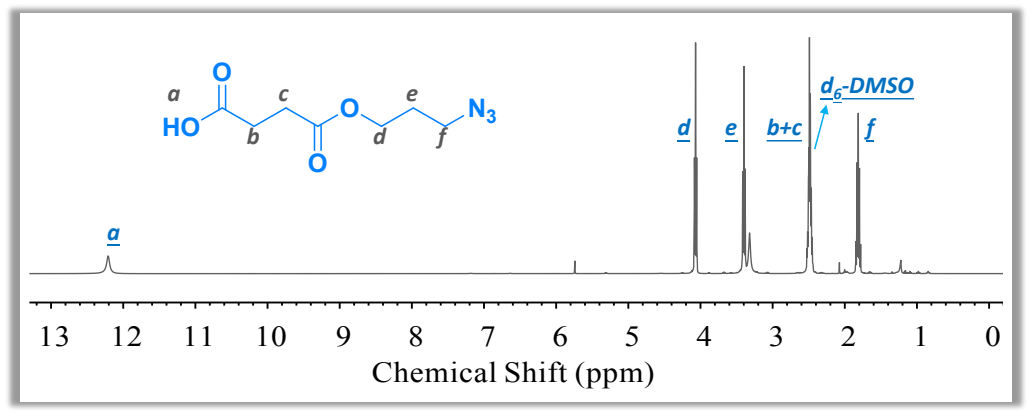

Figure S1. ${ }^{1} \mathrm{H}$ NMR spectrum of $\mathrm{N}_{3}-\mathrm{COOH}$.

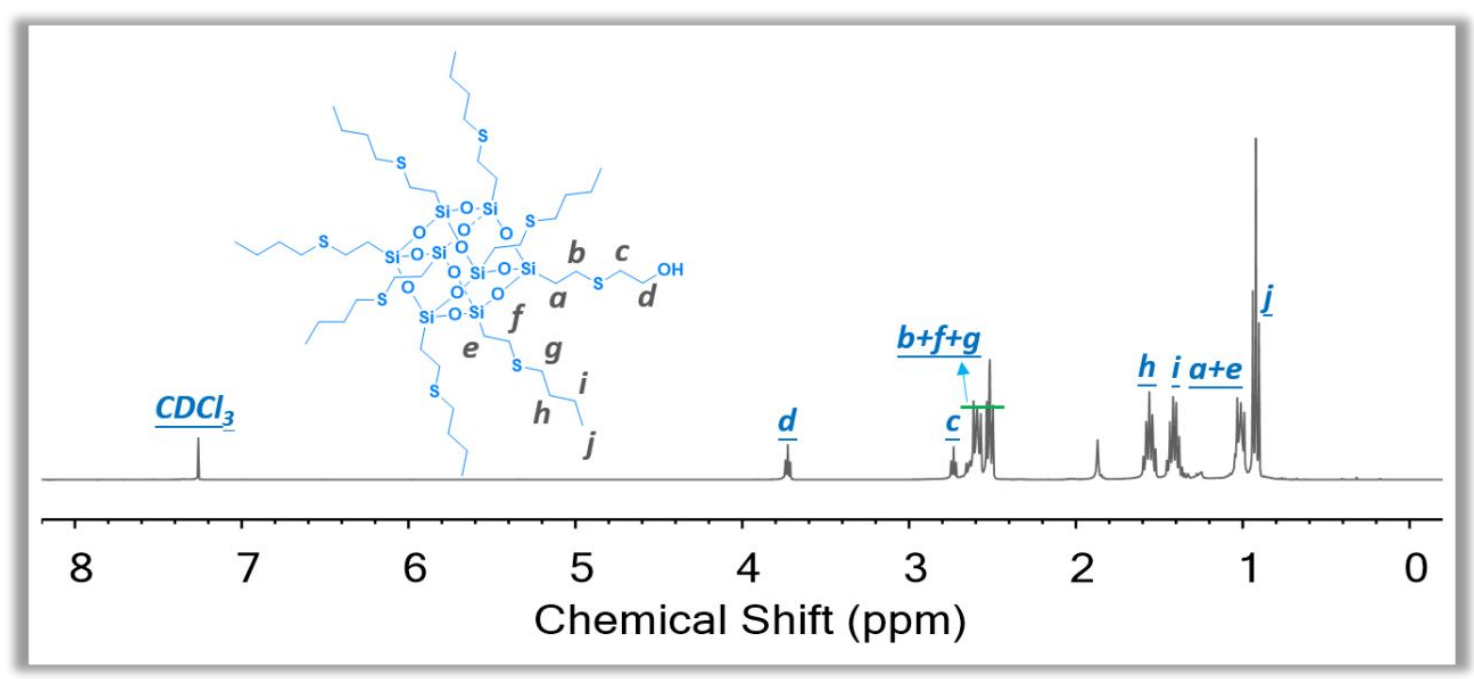

Figure S2. ${ }^{1} \mathrm{H}$ NMR spectrum of CPOSS-OH. 


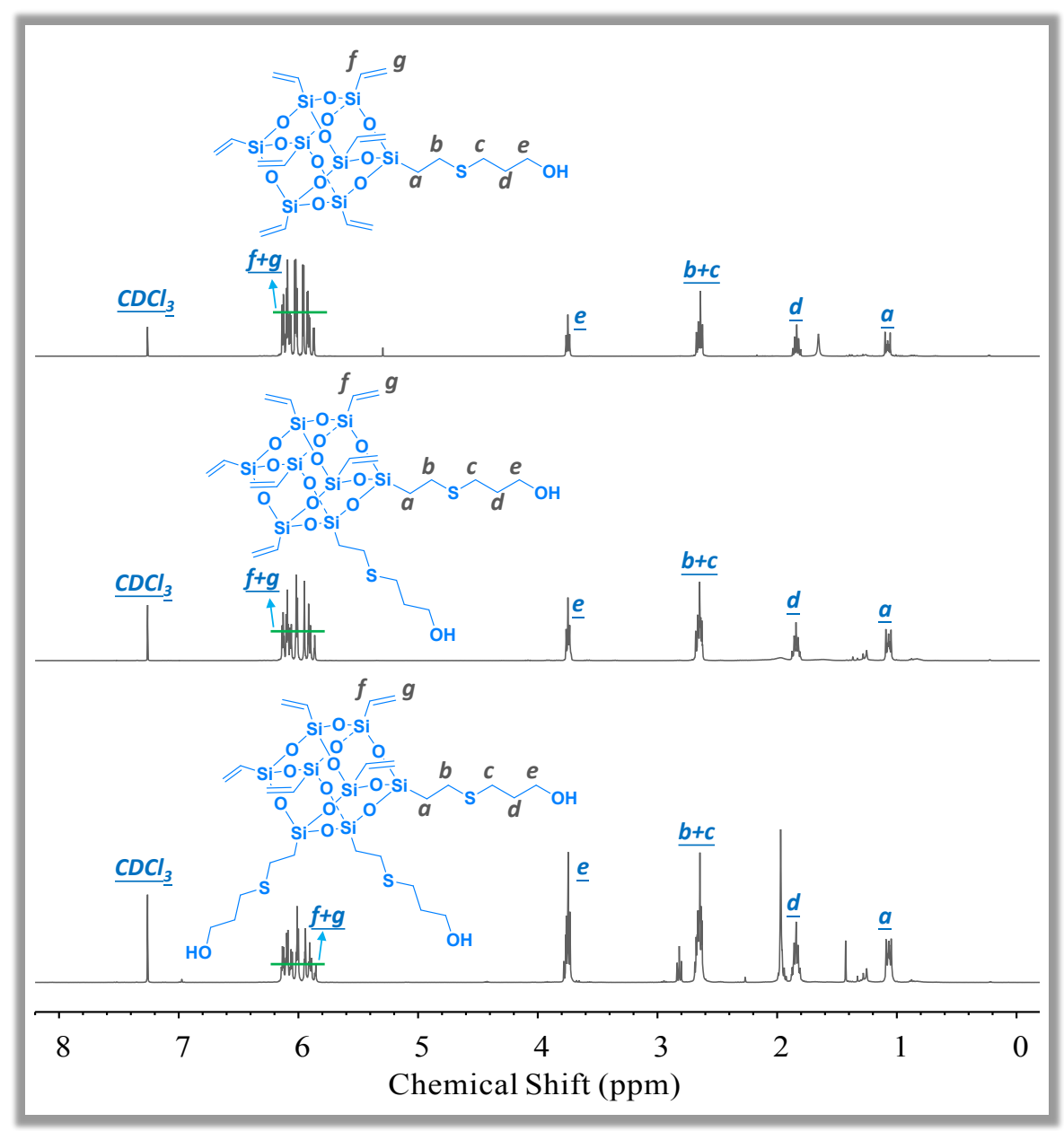

Figure S3. ${ }^{1} \mathrm{H}$ NMR spectrum of $\mathrm{V}_{(8-n)} \mathrm{POSS}(\mathrm{OH})_{n}(n=1,2,3)$ isomers.

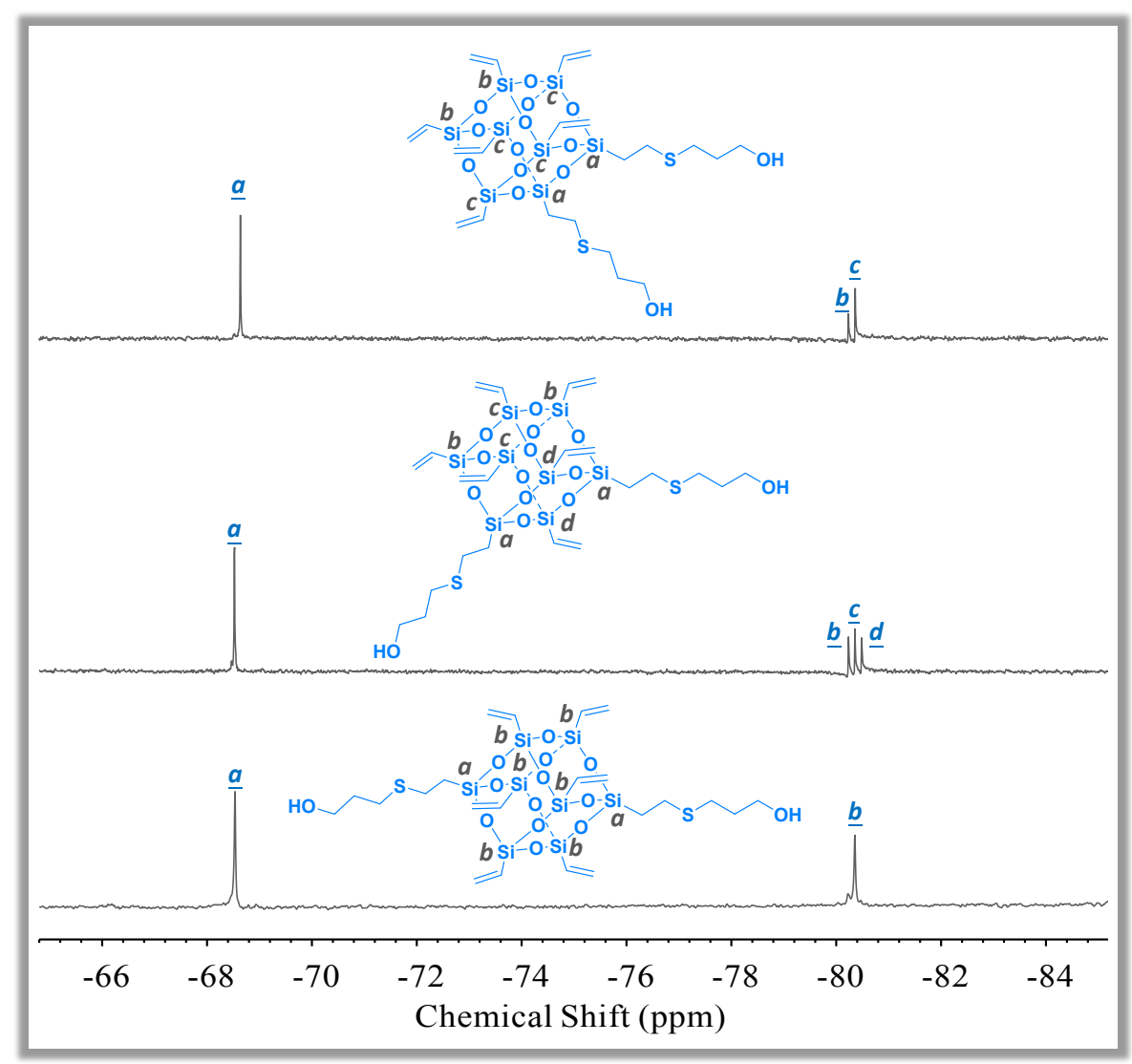

Figure S4. ${ }^{29} \mathrm{Si}$ NMR spectrum of $\mathrm{V}_{6} \mathrm{POSS}(\mathrm{OH})_{2}$ isomers. 


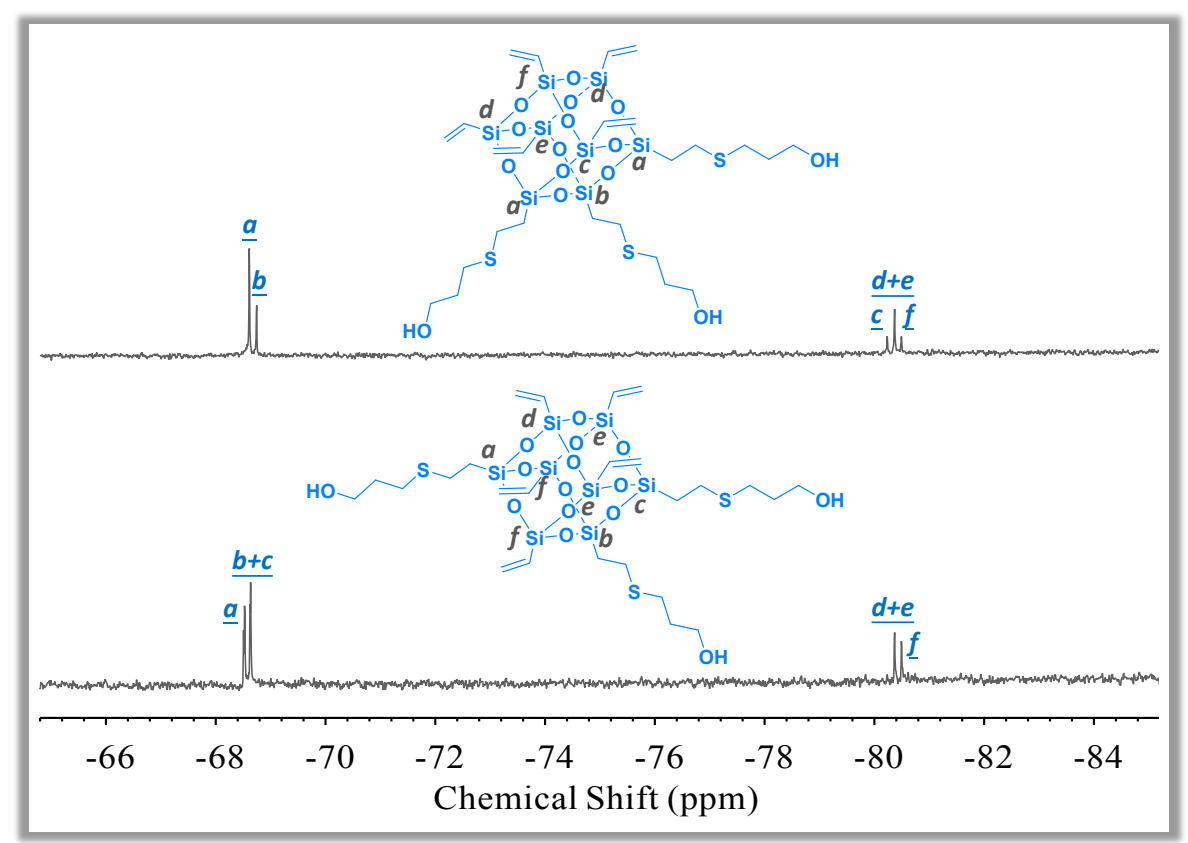

Figure S5. ${ }^{29} \mathrm{Si}$ NMR spectrum of $\mathrm{V}_{5} \mathrm{POSS}(\mathrm{OH})_{3}$ isomers.

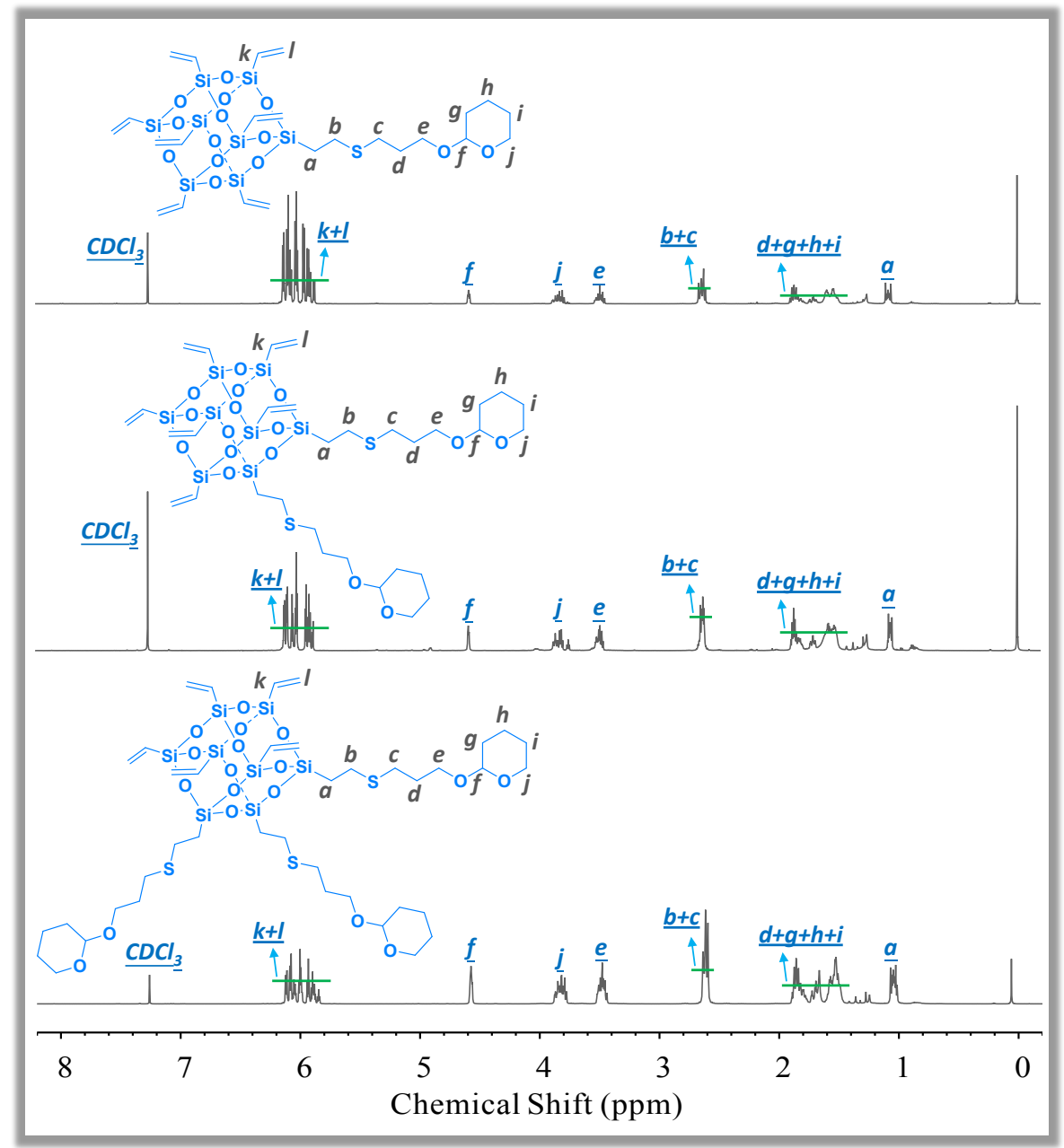

Figure S6. ${ }^{1} \mathrm{H}$ NMR spectra of $\mathrm{V}_{(8-n)} \operatorname{POSS}(\mathrm{THP})_{n}(n=1,2,3)$ isomers. 

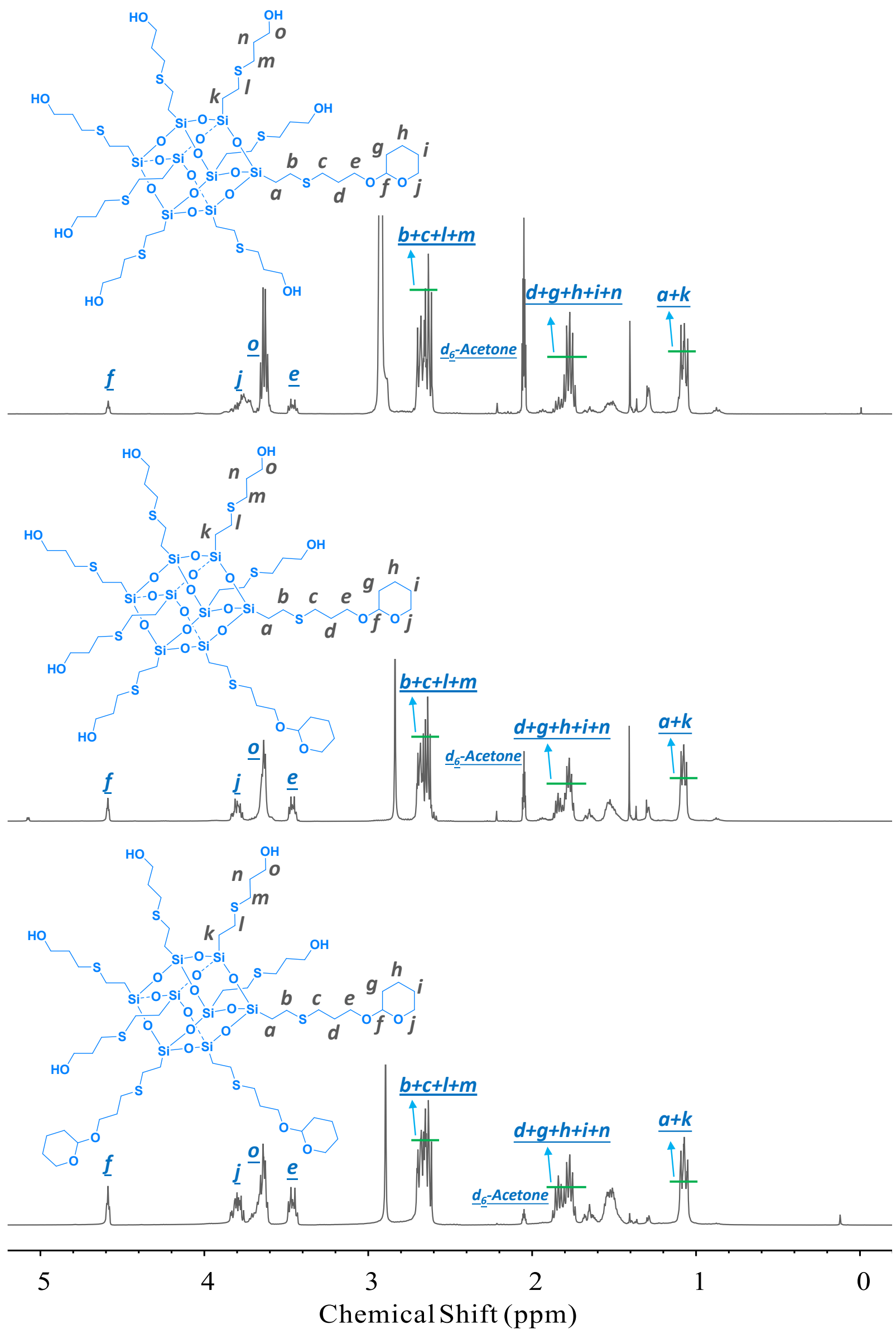

Figure S7. ${ }^{1} \mathrm{H}$ NMR spectra of $\mathrm{HO}_{(8-n)} \mathrm{POSS}(\mathrm{THP})_{n}(\mathrm{n}=1,2,3)$ isomers. 

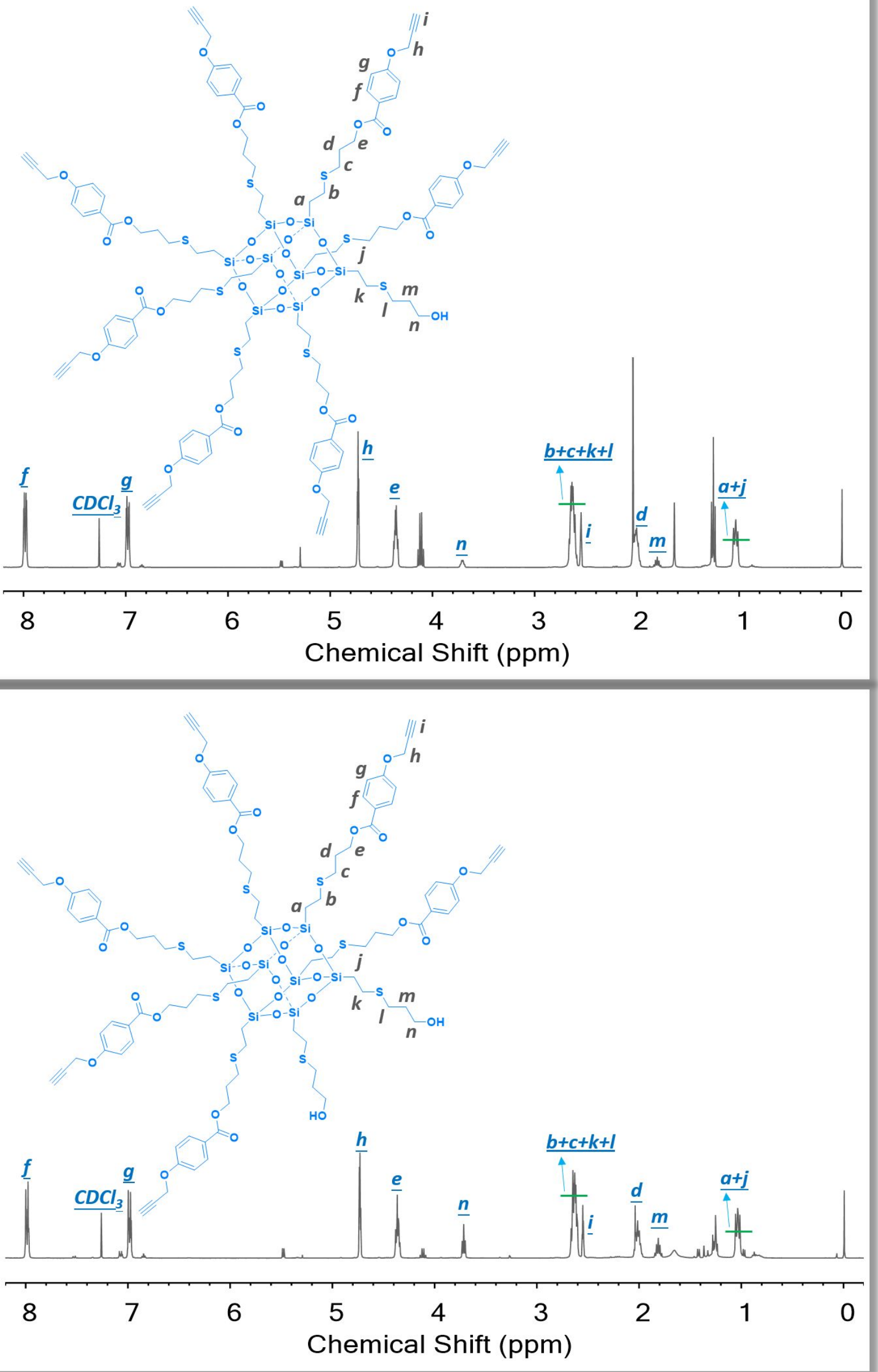

Figure S8. ${ }^{1} \mathrm{H}$ NMR spectra of $(\mathrm{yne})_{(8-n)}(\mathrm{POSS})(\mathrm{OH})_{n}(n=1,2)$ isomers. 


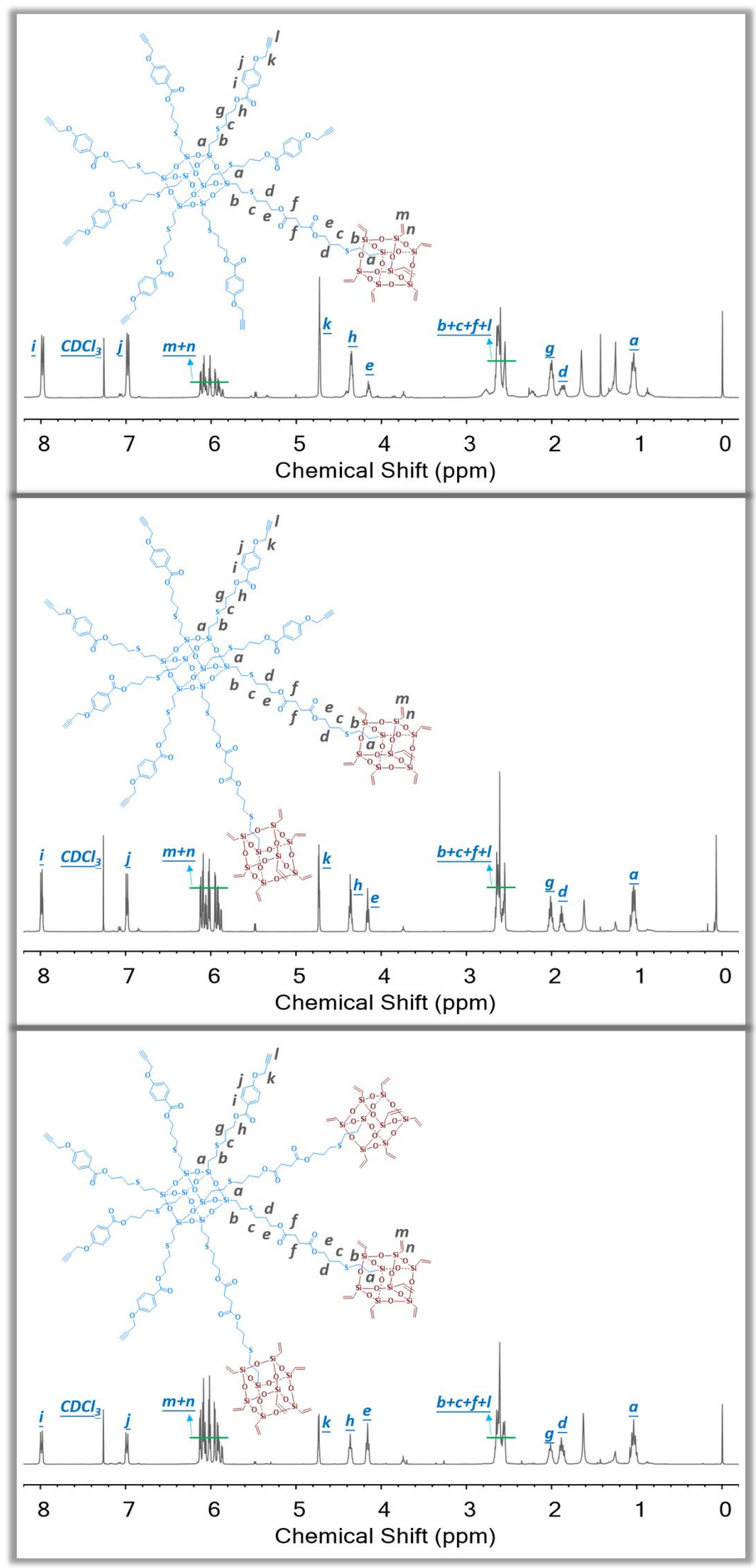

Figure S9. ${ }^{1} \mathrm{H}$ NMR spectra of $(\mathrm{yne})_{(8-n)}(\mathrm{POSS})(\operatorname{VPOSS})_{n}(n=1,2,3)$ isomers. 


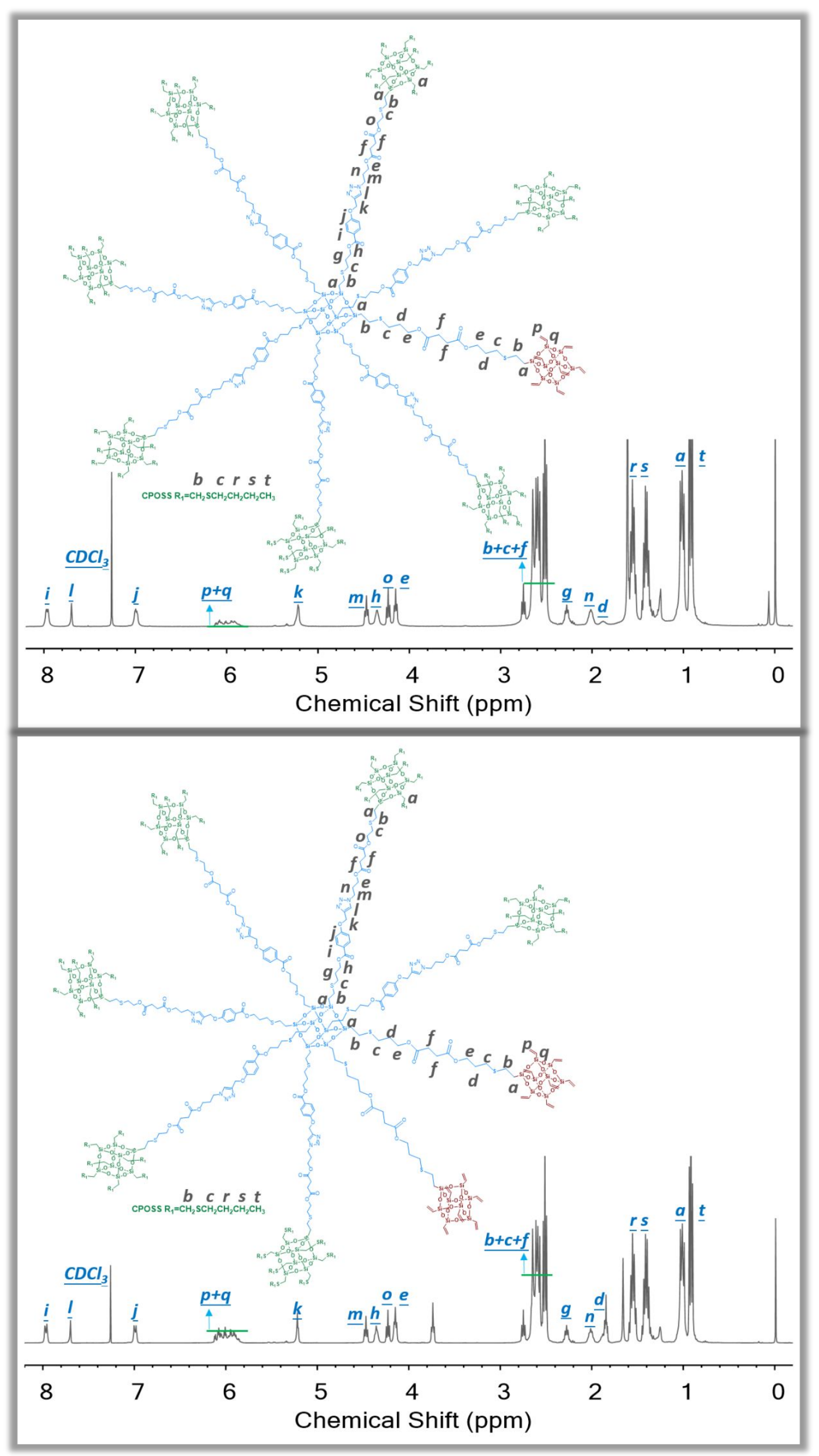

Figure S10. ${ }^{1} \mathrm{H}$ NMR spectra of $(\mathrm{CPOSS})_{(8-n)}(\operatorname{POSS})(\operatorname{VPOSS})_{n}(n=1,2)$ isomers. 


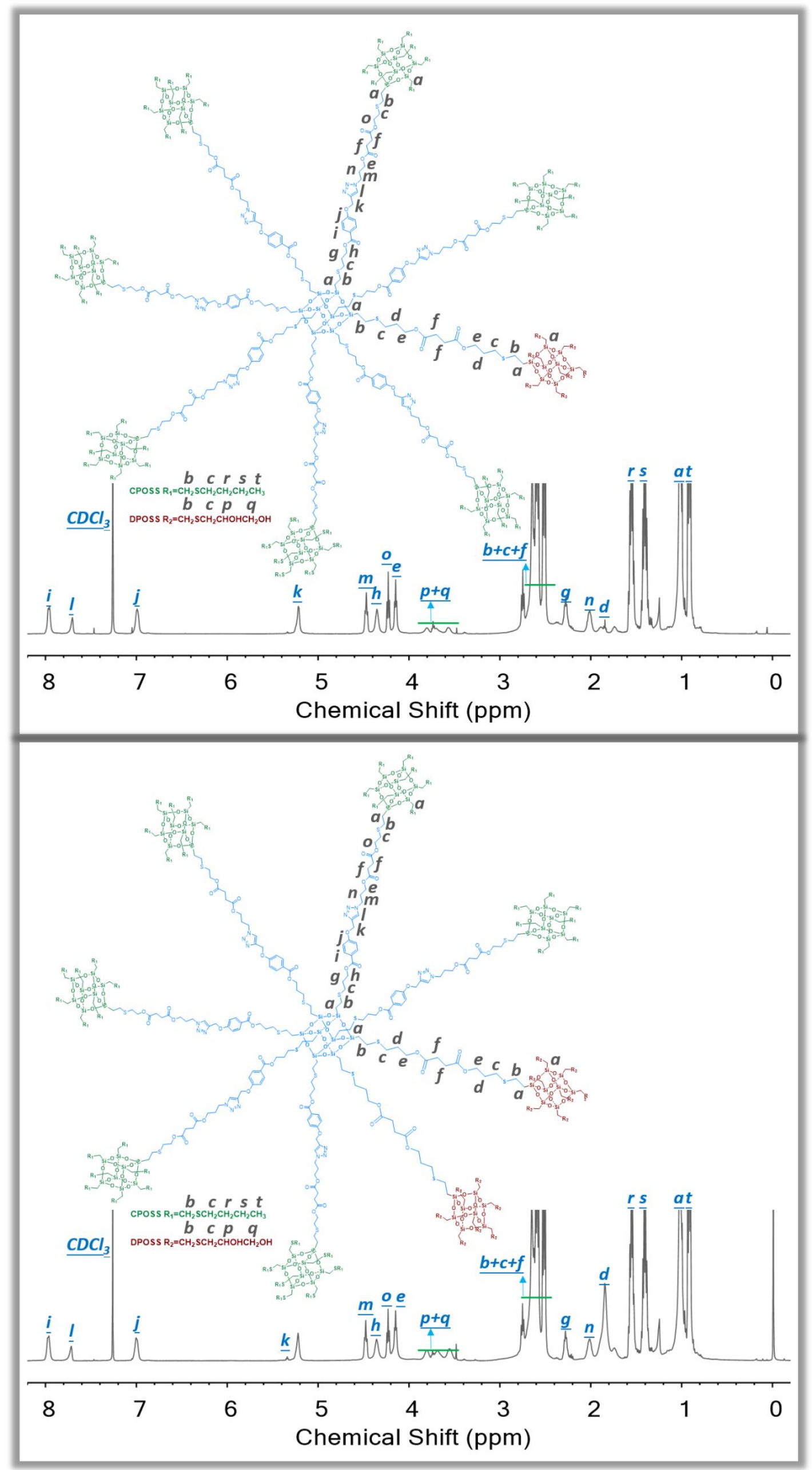

Figure S11. ${ }^{1} \mathrm{H}$ NMR spectra of $(\mathrm{CPOSS})_{(8-n)}(\operatorname{POSS})(\mathrm{DPOSS})_{n}(n=1,2)$ isomers. 

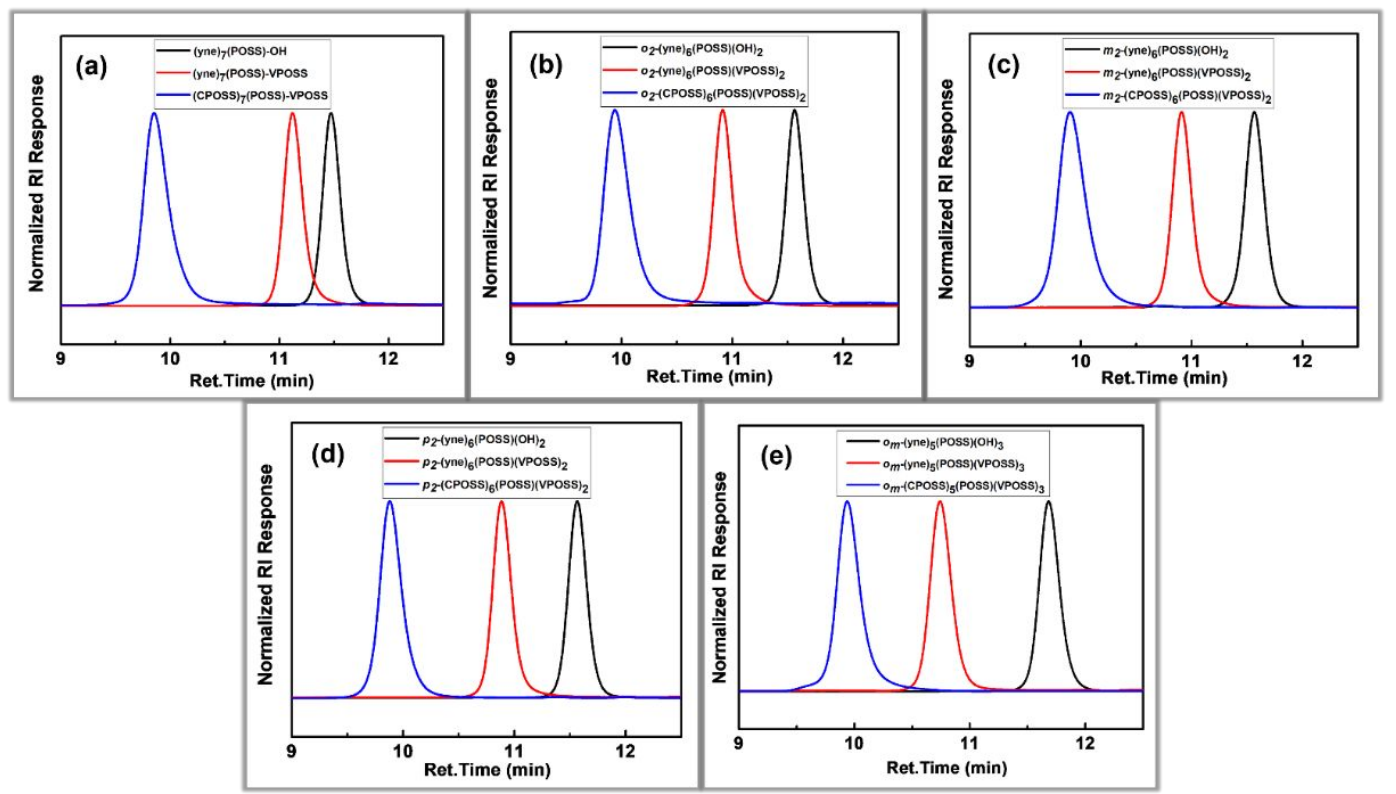

Figure S12. GPC traces of related precursors/ intermediates: (a) 7C1D, (b) $o_{2}-6 \mathrm{C} 2 \mathrm{D}$, (c) $m_{2}-6 \mathrm{C} 2 \mathrm{D}$, (d) $p_{2}-6 \mathrm{C} 2 \mathrm{D}$, (e) $o_{m}-5 \mathrm{C} 3 \mathrm{D}$.

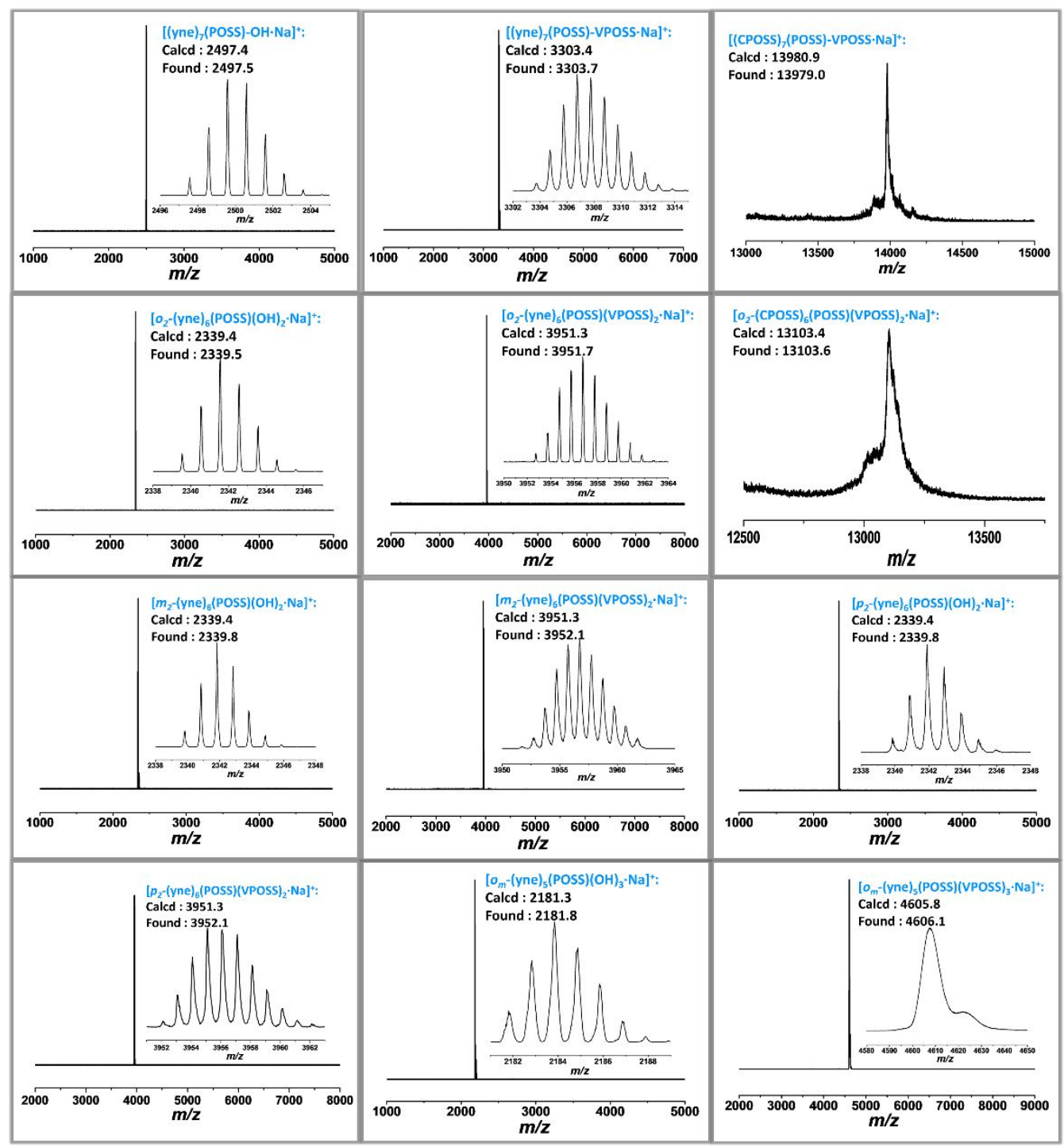

Figure S13. MALDI-TOF-MS of related precursors/ intermediates. 


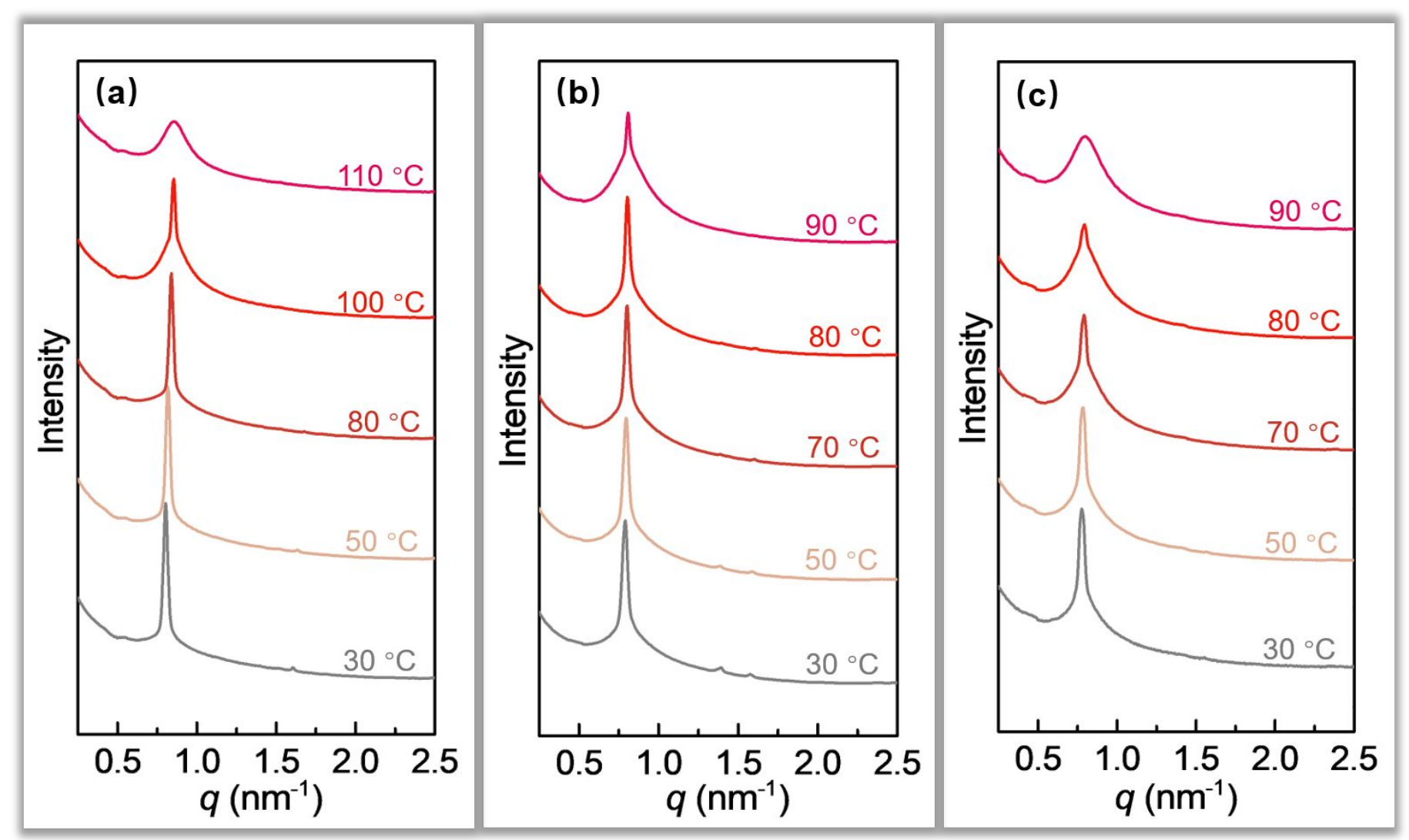

Figure S14. In-situ temperature SAXS scan patterns for heating process: (a) $m_{2}-6 \mathrm{C} 2 \mathrm{D}$, (b) $p_{2}-6 \mathrm{C} 2 \mathrm{D}$, (c) $o_{m}-5 \mathrm{C} 3 \mathrm{D}$.

Table S1. Experimentally observed $\left(q_{\mathrm{obs}}\right)$ and calculated $\left(q_{\mathrm{cal}}\right)$ from the $o_{2}-6 \mathrm{C} 2 \mathrm{D}$ SAXS pattern $(a=33.59 \mathrm{~nm}, c=17.77$ $\mathrm{nm})$

\begin{tabular}{ccc}
\hline$q_{\mathrm{obs}}(\mathrm{nm})^{-1}$ & $q_{\mathrm{cal}}(\mathrm{nm})^{-1}$ & $(\mathrm{~h} \mathrm{k} \mathrm{1})$ \\
\hline 0.592 & 0.592 & 310 \\
0.637 & 0.636 & 221 \\
0.689 & 0.689 & 311 \\
0.707 & 0.707 & 002 \\
0.771 & 0.771 & 410 \\
0.794 & 0.794 & 330 \\
0.794 & 0.800 & 202 \\
0.822 & 0.822 & 212 \\
0.849 & 0.848 & 411 \\
0.867 & 0.869 & 331 \\
0.922 & 0.922 & 312 \\
0.977 & 0.977 & 322 \\
0.100 & 0.100 & 501 \\
0.100 & 0.100 & 431 \\
1.018 & 1.017 & 511 \\
\hline
\end{tabular}


Table S2. Calculation of volume fraction and density of molecular patchy clusters

\begin{tabular}{ccccc}
\hline Sample & $\begin{array}{c}\text { Molecular } \\
\text { weight }\end{array}$ & $\begin{array}{c}\rho \\
\left(\mathrm{g} / \mathrm{cm}^{3}\right)^{a}\end{array}$ & $\begin{array}{c}V_{f}{ }^{C P O S S} \\
(\%)^{b}\end{array}$ & $\begin{array}{c}V_{f}{ }^{\text {DPOSS }} \\
(\%)^{c}\end{array}$ \\
\hline 7C1D & 14721.1 & 1.16 & 92.5 & 7.5 \\
$o_{2}$-6C2D & 14601.6 & 1.19 & 84.5 & 15.5 \\
$m_{2}$-6C2D & 14601.6 & 1.19 & 84.5 & 15.5 \\
$p_{2}$-6C2D & 14601.6 & 1.19 & 84.5 & 15.5 \\
$o_{3}$-5C3D & 14482.2 & 1.21 & 76.1 & 23.9 \\
$o_{m}-5 \mathrm{C} 3 \mathrm{D}$ & 14482.2 & 1.21 & 76.1 & 23.9 \\
\hline
\end{tabular}

${ }^{a}$ The density of these samples calculated approximate to the equation $\rho=V_{f}^{\text {CPOSS }} \cdot \rho_{\text {CPOSS }}+V_{f}^{\text {PPOSS }} \cdot \rho_{\text {DPOss. }}{ }^{\text {b } V o l u m e ~ f r a c t i o n ~ o f ~}$ CPOSS is calculated by the equation $V_{f}{ }^{\mathrm{CPOSS}}=\mathrm{M}_{\mathrm{CPOSS}} / \rho_{\mathrm{CPOSS}} /\left(\mathrm{M}_{\mathrm{CPOSS}} / \rho_{\mathrm{CPOSS}}+\mathrm{M}_{\mathrm{DPOSS}} / \rho_{\mathrm{DPOSS}}\right),{ }^{c}$ Volume fraction of DPOSS is

calculated by the equation $V_{f}$ DPOSS $=\mathrm{M}_{\mathrm{DPOSS}} / \rho_{\mathrm{DPOSS}} /\left(\mathrm{M}_{\mathrm{CPOSS}} / \rho_{\mathrm{CPOSS}}+\mathrm{M}_{\mathrm{DPOSS}} / \rho_{\mathrm{DPOSS}}\right)$. The density values approximate to 1.13 $\mathrm{g} / \mathrm{cm}^{3}$ for CPOSS, $1.43 \mathrm{~g} / \mathrm{cm}^{3}$ for DPOSS according to the reported work.

\section{References}

1. Tan, R.; Zhou, D.; Liu, B.; Sun, Y.; Liu, X.; Ma, Z.; Kong, D.; He, J.; Zhang, Z.; Dong, X. H. Precise Modulation of Molecular Weight Distribution for Structural Engineering. Chem. Sci. 2019, 10, 10698-10705.

2. Willersinn, J.; Bogomolova, A.; Cabré, M. B.; Schmidt, B. V. K. J. Vesicles of Double Hydrophilic Pullulan and Poly(acrylamide) Block Copolymers: A Combination of Synthetic- and Bio-Derived Blocks. Polym. Chem. 2017, 8, 1244-1254.

3. Liu, G.; Feng, X.; Lang, K.; Zhang, R.; Guo, D.; Yang, S.; Cheng, S. Z. D. Dynamics of Shape-Persistent Giant Molecules: Zimm-Like Melt, Elastic Plateau, and Cooperative Glass-Like. Macromolecules 2017, 50, $6637-6646$.

4. Liu, Y.; Liu, G.; Zhang, W.; Du, C.; Wesdemiotis, C.; Cheng, S. Z. D. Cooperative Soft-Cluster Glass in Giant Molecular Clusters. Macromolecules 2019, 52, 4341-4348. 